\title{
Effect of magnetic field on Newtonian fluid sandwiched between non-Newtonian fluids through porous cylindrical shells
}

Deepak Kumar Maurya ( $\sim$ deepak893395@gmail.com )

Veer Bahadur Singh Purvanchal University https://orcid.org/0000-0003-2228-3606

Satya Deo

University of Allahabad

\section{Research Article}

Keywords: Micropolar fluids, Couple stress, Hartmann number, Flow rate

Posted Date: April 29th, 2021

DOI: https://doi.org/10.21203/rs.3.rs-470418/v1

License: (c) (1) This work is licensed under a Creative Commons Attribution 4.0 International License.

Read Full License

Version of Record: A version of this preprint was published at Special Topics \&amp; Reviews in Porous Media: An International Journal on January 1st, 2022. See the published version at https://doi.org/10.1615/SpecialTopicsRevPorousMedia.2022039882. 


\title{
Effect of magnetic field on Newtonian fluid sandwiched between non-Newtonian fluids through porous cylindrical shells
}

\author{
Deepak Kumar Maurya ${ }^{1,2, a}$ and Satya Deo ${ }^{1, b}$ \\ ${ }^{1}$ Department of Mathematics, University of Allahabad, Prayagraj, 211002 (U.P.) India \\ ${ }^{2}$ Department of Mathematics, Prof. Rajendra Singh (Rajju Bhaiya) Institute of Physical \\ Sciences for Study and Research, V. B. S. Purvanchal University, Jaunpur, 222003 (U.P.) India
}

\begin{abstract}
The present work deals with the influence of magnetic field on Newtonian fluid sandwiched between two porous cylindrical pipes which are filled with micropolar fluids. Fluid motion is occurring along $z^{*}$-axis and applied magnetic field is taken in the direction perpendicular to the direction of fluid motion. On applying appropriate boundary conditions, velocity profiles, microrotations, flow rate and shear stresses are obtained for the corresponding fluid regions. The graphs for volumetric flow rate and fluid velocity are plotted and discussed for different values of micropolar parameter, couple stress parameter, porosity, viscosity ratio parameter, Hartmann number, conductivity ratio parameters and Darcy numbers.
\end{abstract}

MSC (2020): 76A05, 76S05, 76W05, 35Q35

Keywords: Micropolar fluids, Couple stress, Hartmann number, Flow rate

\section{Introduction}

Micropolar fluids [1] consist of rigid, randomly oriented cylindrical/spherical particles, having microstructure and belong to a class of fluids with non-symmetric stress tensor. Eringen [2] developed theories on micropolar fluids consisting the effects of couple stresses and microstructure systematically. Equations of motion are derived from the laws of conservation of linear and angular momenta, described in the classical book of Nowacki [3]. Flows of micropolar liquids can exhibit many effects that are not possible in non-polar Stokesian fluids [4]. Creeping flow of a rotating porous cylinder through a micropolar fluid is reported by Moosaie and Atefi [5]. Sherief et al. [6] investigated the micropolar fluid flows (both parallel and perpendicular) between slip cylinder and coaxial cylindrical

\footnotetext{
adeepak893395@gmail.com (corresponding author)

bsd_mathau@yahoo.co.in
} 
shell. Mathematical models for flows of a non-Newtonian liquid have been reviewed by Khanukaeva and Filippov [7]. The micropolar fluid flow through a swarm of cylindrical particles with porous layer, reported by Khanukaeva et al. [8]. In the presence of magnetic field, the micropolar fluid flow through the membrane is observed [9]. Yadav and Verma [10] investigated the fluid motion of two immiscible fluids (Newtonian and non-Newtonian) through a porous cylindrical pipe. Deo et al. [11] studied the micropolar fluid motion moving through a cylindrical tube in presence of the magnetic field which is coated with porous layer. Yadav et al. [12] formulated the mathematical model for Newtonian and non-Newtonian fluids through concentric pipes having porous space of different permeability. Maurya and Deo [13] reported the stream function solution of the Brinkman/Stokes equations in parabolic cylindrical coordinates.

The porosity [14] of a porous medium is defined as the fraction of the total volume of the medium that is occupied by void space. Brinkman [15] investigated the viscous force, exerted by a flowing fluid on a dense swarm of particles. Numerical results for flow between two parallel plates show that the entrance length decreases linearly as the Darcy number decreases, reported by Kaviany [16]. Tiwari and Deo [17] studied pulsatile flow of Newtonian fluid in a cylindrical pipe. Madasu and Bucha [18] observed the effects of magnetic field on fluid flow through a porous cylindrical shell. In cylindrical polar coordinates, generalized version of the stream function solution of Brinkman equation is obtained [19]. Recently, Maurya et al. [20] investigated the Stokes flow of non-Newtonian fluid flowing through a porous cylinder and presented a comparison of flow pattern for two types of boundary value problems.

In this study, we have discussed the effects of magnetic field on the Newtonian fluid flowing through a porous cylindrical shell, sandwiched between two porous cylindrical shells which are filled with non-Newtonian fluids (e.g. micropolar fluids). The direction of fluid flow is taken along $z^{*}$-axis and the uniform magnetic field is applied in the direction perpendicular to the direction of fluid motion. For the corresponding fluid flow regions, velocity profiles, flow rate and shear stresses are obtained by applying suitable boundary conditions. The volumetric flow rate and fluid velocity are plotted and discussed for different values of micropolar parameter, couple stress parameter, porosity, viscosity ratio 
parameter, Hartmann number, conductivity ratio parameters and Darcy numbers.

\section{Mathematical Formulation of the Problem}

In the presence of uniform magnetic field $B^{*}$, let us consider a steady, incompressible, electrically conducting, Newtonian and micropolar fluids, moving with uniform velocity $U^{*}$ along the $z^{*}$-axis of coaxial porous cylinders. The uniform magnetic field is taken in the direction perpendicular to the direction of the fluid flow. The present research model exhibits the sandwiching of Newtonian fluid between the micropolar fluids through two porous coaxial cylindrical shells (Fig. 1). Due to the constant pressure gradient along the direction of the fluid motion, Hagen-Poiseuille flow is used. The Nowacki's governing equations of micropolar fluid is used for non-Newtonian fluids, and Brinkman type equation has been used for the Newtonian fluid flowing through the porous medium. The micropolar fluid of permeability $k_{1}{ }^{*}$ is flowing in the inner cylindrical shell whose radius is $a^{*}$, the middle porous cylindrical shell of radius $b^{*}$ is filled with Newtonian fluid of permeability $k_{2}{ }^{*}$ and the upper porous cylindrical region is of radius $c^{*}$ which is filled with micropolar fluid of permeability $k_{3}{ }^{*}$.

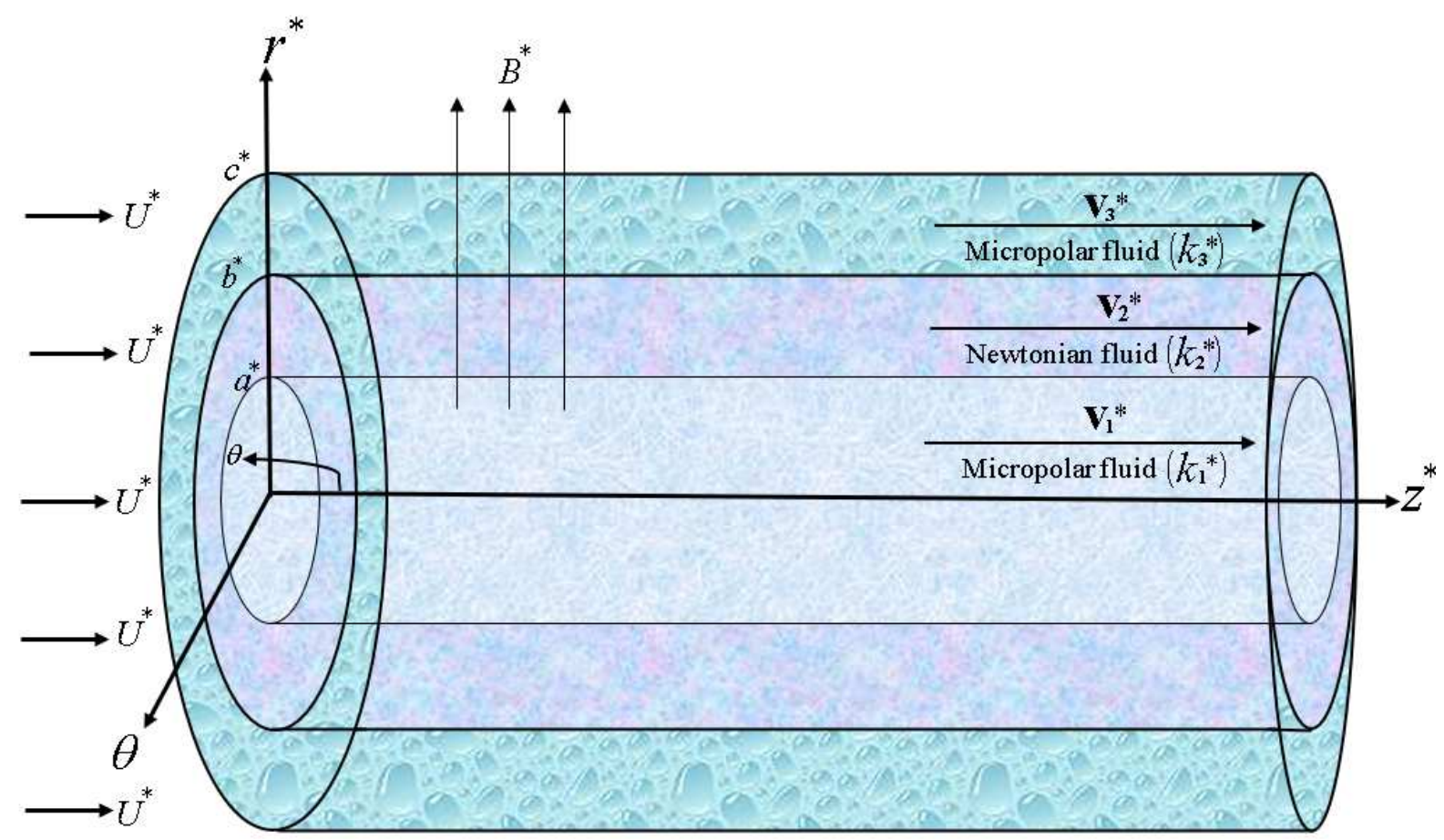

Fig. 1: Schematic diagram of the problem 
In the absense of magnetic field, body forces and body couples, governing equations of an incompressible steady micropolar fluid flow in the Eringen approach, are given by

$$
\begin{gathered}
\nabla^{*} \cdot \mathbf{v}^{*}=0 \\
\left(\frac{\mu_{e}^{*}+\kappa_{e}^{*}}{k^{*}}\right) \mathbf{v}^{*}+\nabla^{*} p^{*}-\frac{\kappa_{e}^{*}}{2 \phi} \nabla^{*} \times \mathbf{w}^{*}+\left(\frac{\mu_{e}^{*}+\kappa_{e}^{*}}{\phi}\right) \nabla^{*} \times \nabla^{*} \times \mathbf{v}^{*}=\mathbf{0}, \\
-2 \kappa_{e}^{*} \mathbf{w}^{*}+\kappa_{e}^{*} \nabla^{*} \times \mathbf{v}^{*}-\chi_{e}^{*} \nabla^{*} \times \nabla^{*} \times \mathbf{w}^{*}+\left(\xi_{e}^{*}+\zeta_{e}^{*}\right) \nabla^{*}\left(\nabla^{*} \cdot \mathbf{w}^{*}\right)=\mathbf{0} .
\end{gathered}
$$

Here, $\phi$ is porosity and $k^{*}$ is permeability of the porous cylindrical shells, and the fluid velocity vector, microrotation vector and fluid pressure, are represented by $\mathbf{v}^{*}, \mathbf{w}^{*}$ and $p^{*}$, respectively. The state variables $\left(\mu_{e}^{*}, \kappa_{e}^{*}\right)$ are used for viscosity coefficients and $\left(\xi_{e}^{*}, \zeta_{e}^{*}, \chi_{e}^{*}\right)$ are taken as angular-viscosity coefficients of the micropolar fluid.

In general, viscosity coefficient $\mu_{e}^{*}$ is not equal to dynamic coefficient of viscosity $\mu^{*}$ of a Newtonian fluid. Redefining the viscosity coefficients using the replacement $\mu_{e}^{*}=\mu^{*}-\kappa^{*}$ and $\kappa_{e}^{*}=2 \kappa^{*}$ on applying Stokes hypothesis. To derive the components of deformation tensors and for reducing the coefficient $\mu_{e}^{*}$ into $\mu^{*}$, Nowacki proposed to take the angular viscosity coefficients as $\chi_{e}^{*}=\delta^{*}+\tau^{*}, \zeta_{e}^{*}=\delta^{*}-\tau^{*}$ and $\xi_{e}^{*}=\xi^{*}$.

Nowacki's governing equations of an incompressible steady micropolar fluid flow through the porous cylindrical shells $\left(0 \leq r^{*} \leq a^{*}\right)$ and $\left(b^{*} \leq r^{*} \leq c^{*}\right)$ in the presence of uniform magnetic field $B^{*}$, are given by

$$
\begin{gathered}
\nabla^{*} \cdot \mathbf{v}_{i}^{*}=0, \\
\left(\frac{\mu^{*}+\kappa^{*}}{k_{i}^{*}}\right) \mathbf{v}_{i}^{*}+\nabla^{*} p^{*}-\left(\frac{\kappa^{*}}{\phi}\right) \nabla^{*} \times \mathbf{w}_{i}^{*}+\left(\frac{\mu^{*}+\kappa^{*}}{\phi}\right) \nabla^{*} \times \nabla^{*} \times \mathbf{v}_{i}^{*}-\mathbf{J}_{i}^{*} \times \mathbf{B}^{*}=\mathbf{0}, \\
-2 \kappa^{*} \mathbf{w}_{i}^{*}+\kappa^{*} \nabla^{*} \times \mathbf{v}_{i}^{*}-\left(\delta^{*}+\tau^{*}\right) \nabla^{*} \times \nabla^{*} \times \mathbf{w}_{i}^{*}+\left(\xi^{*}+\delta^{*}-\tau^{*}\right) \nabla^{*}\left(\nabla^{*} \cdot \mathbf{w}_{i}^{*}\right)=\mathbf{0},
\end{gathered}
$$

where, $\mathbf{v}_{i}^{*}, \mathbf{w}_{i}^{*}$ are representing the velocity vectors and microrotation vectors of micropolar fluid for inner porous cylindrical shell $(i=1)$ and outer porous cylindrical shell $(i=3)$, at any point $\left(r^{*}, \theta, z^{*}\right)$, respectively. The material's constants $\left(\mu^{*}, \kappa^{*}\right)$ are viscosity coefficients and $\left(\xi^{*}, \delta^{*}, \tau^{*}\right)$ are gyro-viscosity coefficients. These viscosity coefficients are related by inequalities:

$$
\mu^{*} \geq 0, \quad \kappa^{*} \geq 0, \quad \delta^{*} \geq 0, \quad 3 \xi^{*}+2 \delta^{*} \geq 0, \quad\left|\delta^{*}-\tau^{*}\right| \leq\left(\delta^{*}+\tau^{*}\right) .
$$

The governing equations for Newtonian fluid through the porous cylindrical shell $\left(a^{*} \leq\right.$ $r^{*} \leq b^{*}$ ), in the presence of uniform magnetic field and in the absense of body forces, are 
given by

$$
\begin{gathered}
\nabla^{*} \cdot \mathbf{v}_{2}^{*}=0 \\
-\nabla^{*} p^{*}-\left(\frac{\mu_{2}^{*}}{k_{2}{ }^{*}}\right) \mathbf{v}_{2}^{*}-\left(\frac{\mu_{2}{ }^{*}}{\phi}\right) \nabla^{*} \times \nabla^{*} \times \mathbf{v}_{2}^{*}+\mathbf{J}_{2}^{*} \times \mathbf{B}^{*}=\mathbf{0},
\end{gathered}
$$

where, $\mathbf{v}_{2}^{*}$ and $\mu_{2}^{*}$ are fluid velocity vector and dynamic viscosity of the Newtonian fluid, respectively. The applied fluid pressure $p^{*}$ is same for both the micropolar and Newtonian fluids. Assuming that the magnetic Reynolds number is negligible and external electric field $\left(\mathbf{E}^{*}\right)$ is absent, then the induced electric current can be neglected. By Ohm's law, $\mathbf{J}_{i}^{*}=\sigma_{i}^{*}\left(\mathbf{E}^{*}+\mathbf{v}_{i}^{*} \times \mathbf{B}^{*}\right)$, where, $\sigma_{i}^{*}(i=1,2,3)$ and $\mathbf{E}^{*}$ stand for electrical conductivity of micropolar fluid and electric field, respectively. Therefore,

$$
\mathbf{J}_{i}^{*} \times \mathbf{B}^{*}=-\sigma_{i}^{*} B^{* 2} \mathbf{v}_{i}^{*}
$$

where, $B^{*}=\left|\mathbf{B}^{*}\right|$, the magnitude of applied uniform magnetic field.

In order to treat governing equations of the micropolar fluid flow problem into dimensionless form, some non-dimensionalising variables are introduced:

$$
\begin{gathered}
\mathbf{v}_{i}=\frac{\mathbf{v}_{i}^{*}}{U^{*}}, \quad \mathbf{w}_{i}=\frac{a^{*} \mathbf{w}_{i}^{*}}{U^{*}}, \quad r=\frac{r^{*}}{a^{*}}, \quad l=\frac{b^{*}}{a^{*}}, \quad m=\frac{c^{*}}{a^{*}}, \quad p=\frac{a^{*} p^{*}}{\mu^{*} U^{*}}, \\
k_{i}=\frac{k_{i}^{*}}{a^{* 2}}, \quad \lambda=\frac{\mu_{2}^{*}}{\mu^{*}}, \quad M^{2}=\frac{\kappa^{*}}{\mu^{*}+\kappa^{*}}, \quad L^{2}=\frac{\delta^{*}+\tau^{*}}{2 \mu^{*} a^{* 2}}, \quad W^{2}=\frac{\xi^{*}+\delta^{*}-\tau^{*}}{2 \mu^{*} a^{* 2}}, \\
\Lambda_{2}=\frac{\sigma_{2}^{*}}{\sigma_{1}^{*}}, \quad \Lambda_{3}=\frac{\sigma_{3}^{*}}{\sigma_{1}^{*}}, \quad H_{1}=B^{*} a^{*} \sqrt{\frac{\sigma_{1}^{*}}{\mu^{*}}}, \quad H_{2}=H_{1} \sqrt{\Lambda_{2}}, \quad H_{3}=H_{1} \sqrt{\Lambda_{3}} .
\end{gathered}
$$

Here, $H_{1}$ is the Hartmann number $\left(0 \leq H_{1}<\infty\right), M$ is micropolar parameter $(0 \leq M<$ $1)$ and $L$ is couple stress parameter $(0 \leq L<\infty)$. The non-dimensional form of the governing equations (4) - (6) for $i=1,3$, are

$$
\begin{gathered}
\nabla \cdot \mathbf{v}_{i}=0 \\
\nabla p+\left(\frac{1}{k_{i}\left(1-M^{2}\right)}+H_{i}^{2}\right) \mathbf{v}_{i}-\left(\frac{M^{2}}{\phi\left(1-M^{2}\right)}\right) \nabla \times \mathbf{w}_{i}+\frac{1}{\phi\left(1-M^{2}\right)} \nabla \times \nabla \times \mathbf{v}_{i}=\mathbf{0}, \\
\mathbf{w}_{i}-\frac{1}{2} \nabla \times \mathbf{v}_{i}+\left(\frac{L^{2}\left(1-M^{2}\right)}{M^{2}}\right) \nabla \times \nabla \times \mathbf{w}_{i}-\left(\frac{W^{2}\left(1-M^{2}\right)}{M^{2}}\right) \nabla\left(\nabla \cdot \mathbf{w}_{i}\right)=\mathbf{0} .
\end{gathered}
$$

Similarly, the field equations (7) - (8) for middle porous cylindrical shell are

$$
\begin{gathered}
\nabla \cdot \mathbf{v}_{2}=0 \\
\nabla p=-\left(\frac{\lambda}{k_{2}}\right) \mathbf{v}_{2}+\left(\frac{\lambda}{\phi}\right) \nabla^{2} \mathbf{v}_{2}-H^{2} \mathbf{v}_{2}
\end{gathered}
$$


For micropolar fluid, the non-dimensional form of shear stresses $\left(T_{r z(i)}\right)$ for $i=1,3$, will be

$$
T_{r z(i)}=\left[\frac{1}{1-M^{2}}\left(\frac{d v_{i}}{d r}\right)+\left(\frac{M^{2}}{1-M^{2}}\right) w_{i}\right] .
$$

For Newtonian fluid, the non-dimensional form of shear stress $\left(T_{r z(2)}\right)$ will be

$$
T_{r z(2)}=\lambda \frac{d v_{2}}{d r}
$$

\section{Analytical solution of the Problem}

\subsection{For Inner and Outer Porous Cylindrical Shells}

Assuming that the fluid velocities $\mathbf{v}_{i}$ and microrotation vectors $\mathbf{w}_{i}$ for Hagen Poiseuille flow of micropolar fluid along the $z$-axis of inner $(i=1)$ and outer $(i=3)$ porous cylindrical shells are $\mathbf{v}_{i}=\left(0,0, v_{i}(r)\right)$ and $\mathbf{w}_{i}=\left(0, w_{i}(r), 0\right)$, respectively. Introducing a differential operator $E^{2}$ as

$$
E^{2}=\frac{1}{r} \frac{d}{d r}\left(r \frac{d}{d r}\right)
$$

Then, governing equations (10) - (12) will take the form

$$
\left(E^{2}-\alpha_{i}^{2}\right)\left(E^{2}-\beta_{i}^{2}\right) v_{i}=-\frac{P \phi M^{2}}{L^{2}}
$$

where,

$$
\begin{aligned}
\alpha_{i}^{2}+\beta_{i}^{2} & =\frac{\left(2-M^{2}\right) M^{2}}{2 L^{2}\left(1-M^{2}\right)}+\frac{\phi}{k_{i}}+\left(1-M^{2}\right) H_{i}{ }^{2} \phi, \\
\alpha_{i}^{2} \beta_{i}^{2} & =\frac{\phi M^{2}}{L^{2}\left(1-M^{2}\right)}\left[\left(1-M^{2}\right) H_{i}{ }^{2}+\frac{1}{k_{i}}\right],
\end{aligned}
$$

and pressure gradient $P=\frac{d p}{d z}$ is taken constant.

The general solution of the equation (17) represent velocity of micropolar fluids and comes out as

$$
v_{i}(r)=A_{i} I_{0}\left(\alpha_{i} r\right)+B_{i} K_{0}\left(\alpha_{i} r\right)+C_{i} I_{0}\left(\beta_{i} r\right)+D_{i} K_{0}\left(\beta_{i} r\right)+\frac{\phi M^{2} P}{\alpha_{i}^{2} \beta_{i}^{2} L^{2}},
$$

where, $A_{i}, B_{i}, C_{i}$, and $D_{i}(i=1,3)$ are arbitrary parameters. 


\subsection{For Middle Porous Cylindrical Shell}

Since the fluid velocity $\mathbf{v}_{2}$ for Hagen-Poiseuille flow along the $z$-axis of the middle porous cylindical region which is filled by Newtonian fluid is $\mathbf{v}_{2}=\left(0,0, v_{2}(r)\right)$. Therefore, equations (13) and (14) will reduce to

$$
\frac{d^{2} v_{2}}{d r^{2}}+\frac{1}{r} \frac{d v_{2}}{d r}-n^{2} v_{2}=\frac{P \phi}{\lambda}
$$

where, $n^{2}=\frac{\phi}{k_{2}}+\left(\frac{\phi}{\lambda}\right) H_{2}^{2}$.

So, the analytical form of Newtonian fluid velocity is:

$$
v_{2}(y)=A_{2} I_{0}(n r)+B_{2} K_{0}(n r)-\frac{P \phi}{\lambda n^{2}}
$$

where, $A_{2}$ and $B_{2}$ are arbitrary parameters.

\section{Determination of Arbitrary Parameters}

To determine the ten parameters $A_{1}, B_{1}, C_{1}, D_{1}, A_{2}, B_{2}, A_{3}, B_{3}, C_{3}, D_{3}$, we are applying the following suitable boundary conditions:

(i) Regularity condition within inner cylinder (i.e. $r=0$ ) implies that,

$$
A_{1}=C_{1}=0 .
$$

(ii) No spin condition at $r=1$ implies that,

$$
w_{1}=0 \text {. }
$$

(iii) Continuity of velocity at $r=1$ implies that,

$$
v_{1}=v_{2} .
$$

(iv) Continuity of shear stress at $r=1$ implies that,

$$
T_{r z(1)}=T_{r z(2)}
$$

(v) Continuity of velocity at $r=l$ implies that,

$$
v_{2}=v_{3}
$$


(vi) Continuity of shear stress at $r=l$ implies that,

$$
T_{r z(2)}=T_{r z(3)}
$$

(vii) No spin condition at $r=l$ implies that,

$$
w_{3}=0
$$

(viii) No slip condition at $r=m$ implies that,

$$
v_{3}=0
$$

(ix) Spin condition at outer surface $r=m$ implies that,

$$
\overrightarrow{w_{3}}=\nabla \times \overrightarrow{v_{3}}
$$

Substituting the boundary conditions (BCs) from equations (21)-(29), we will obtain a system of linear equations for the arbitrary parameters occuring in the expressions of velocities $v_{1}, v_{2}, v_{3}$. All parameters have been evaluated uniquely with the utilization of MATHEMATICA software. Due to burdensome expressions of these parameters, we refrain the values of these parameters from the text of manuscript.

\section{Evaluation of flow rate}

The flow rate through cylindrical shells $(0<r<m)$ can be evaluated using the formula:

$$
Q=\int_{0}^{1} v_{1} r d r+\int_{1}^{l} v_{2} r d r+\int_{l}^{m} v_{3} r d r
$$

Substituting the analytical expressions of fluid velocities from equations (18) and (20) in the equation (30), we are capable to determine the mathematical expression for volumetric flow rate of Newtonian-micropolar fluid flow. Therefore, analytical expression of the volumetric flow rate comes out as:

$$
\begin{gathered}
Q=\pi\left[\frac{1}{\xi_{3}}\left(2\left(A_{3}\left(m I_{1}\left(m \xi_{3}\right)-l I_{1}\left(l \xi_{3}\right)\right)+B_{3}\left(l K_{1}\left(l \xi_{3}\right)-m K_{1}\left(m \xi_{3}\right)\right)\right)\right)+A_{10} \tilde{F}_{1}\left(; 2 ; \frac{\xi_{1}^{2}}{4}\right)\right. \\
-\frac{2 A_{2}\left(I_{1}(n)-l I_{1}(l n)\right)}{n}+\frac{2 B_{2}\left(K_{1}(n)-l K_{1}(l n)\right)}{n}+\frac{1}{\zeta_{3}}\left(2 \left(C _ { 3 } \left(m I_{1}\left(m \zeta_{3}\right)\right.\right.\right. \\
\left.\left.\left.-l I_{1}\left(l \zeta_{3}\right)\right)+F_{3}\left(l K_{1}\left(l \zeta_{3}\right)-m K_{1}\left(m \zeta_{3}\right)\right)\right)\right)+C_{10} \tilde{F}_{1}\left(; 2 ; \frac{\zeta_{1}^{2}}{4}\right) \\
\left.+\frac{M^{2} P \phi\left(m^{2}-l^{2}\right)}{\zeta_{3}^{2} L^{2} \xi_{3}^{2}}-\frac{\left(l^{2}-1\right) P \phi}{\lambda n^{2}}+\frac{M^{2} P \phi}{\zeta_{1}^{2} L^{2} \xi_{1}^{2}}\right], \quad \text { (31) }
\end{gathered}
$$


where $\tilde{F}_{1}(; ;)$ is regularized hypergeometric function.

\subsection{Discussion of flow rate with variation of flow parameters:}

Volumetric flow rate $Q$ versus parameter $l(1 \leq l \leq 3)$ is plotted and discussed its graphical behaviors for various values of flow parameters. These flow parameters are well known micropolar parameter $(M)$, porosity $(\phi)$, viscosity ratio $(\lambda)$, Hartmann number $\left(H_{1}\right)$, conductivity ratio parameters $\left(\Lambda_{2}, \Lambda_{3}\right)$, Darcy numbers $\left(k_{1}, k_{2}, k_{3}\right)$. For each case of flow rate, some flow parameters are taken fixed and these are: $L=2, m=5$, and $P=-3$.

\subsubsection{Effect of micropolar parameter $(\mathrm{M})$}

The effects of flow parameter $(M=0.4,0.7,0.8)$ on flow rate are shown in figure 2 . Viscosity ratio parameter $(\lambda=0.2)$, porosity $\phi=0.5$, Hartmann number $\left(H_{1}=1.5\right)$, Darcy numbers $\left(k_{1}=0.3, k_{2}=4, k_{3}=5\right)$ and conductivity ratio parameters $\left(\Lambda_{2}=\right.$ $\left.0.5, \Lambda_{3}=0.25\right)$ are used for plotting. On observation of the graphical behavior of volumetric flow rate, we conclude that volumetric flow rate decreases whenever the flow parameter (i.e. micropolar parameter) increases.

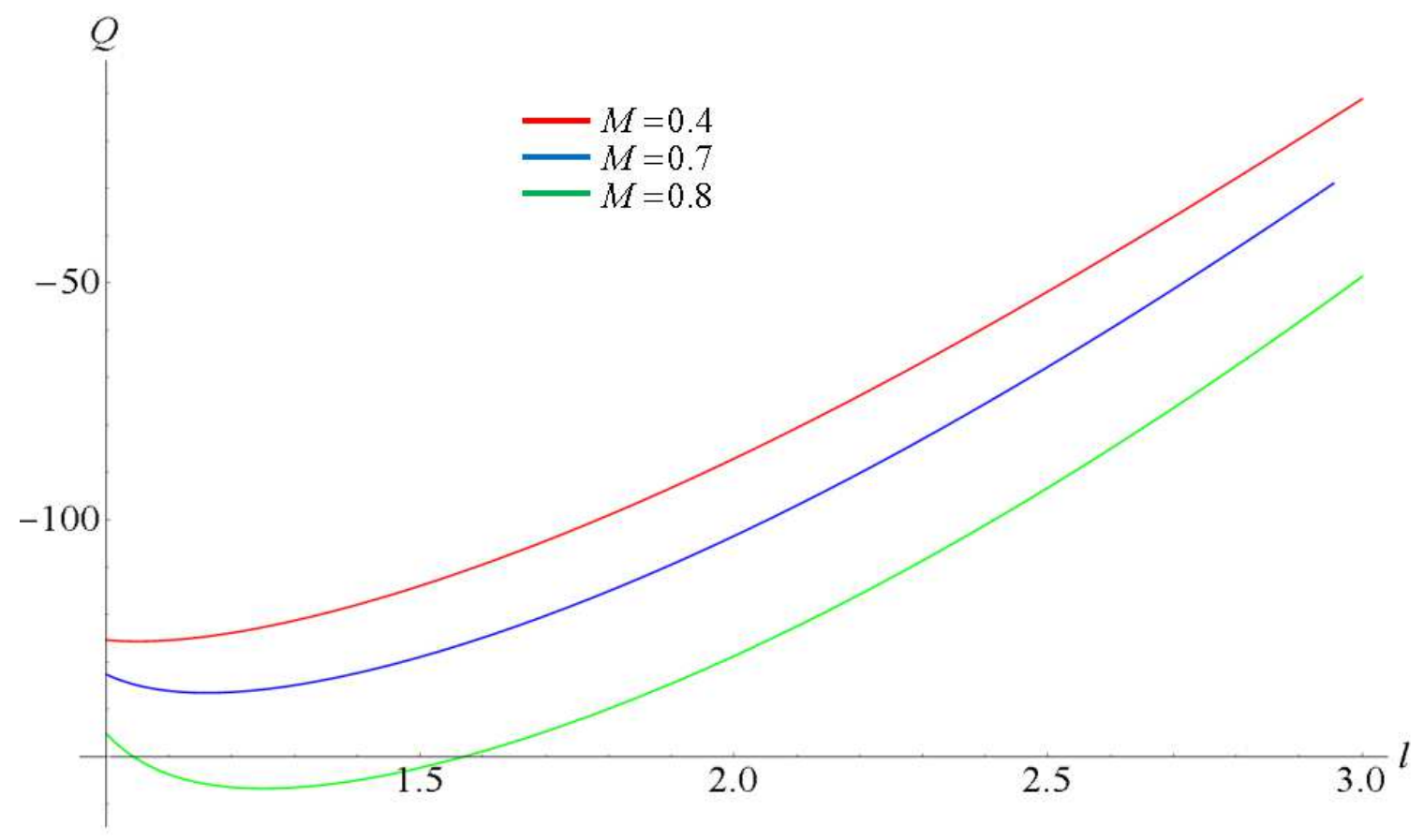

Fig. 2: Variation of flow rate with micropolar parameter $M$. 


\subsubsection{Effect of porosity $\phi$}

Figure 3 exhibits the graphical nature of the flow rate for different values of non-dimensional parameter porosity. Values of other flow parameters are taken as follows: $M=0.2, \lambda=$ $0.2, k_{1}=0.3, k_{2}=4, k_{3}=5, H_{1}=1.5, \Lambda_{2}=0.5, \Lambda_{3}=0.25$, along with the value of porosity is taken as $0.2,0.4,0.6$. The present investigation offers that the flow rate varies smoothly, and converges with the variation of large values of parameter $l$. Also, we obtain that volumetric flow rate decreases as the porosity increases i.e., voidness of the space with respect to total volume of the porous medium increases.

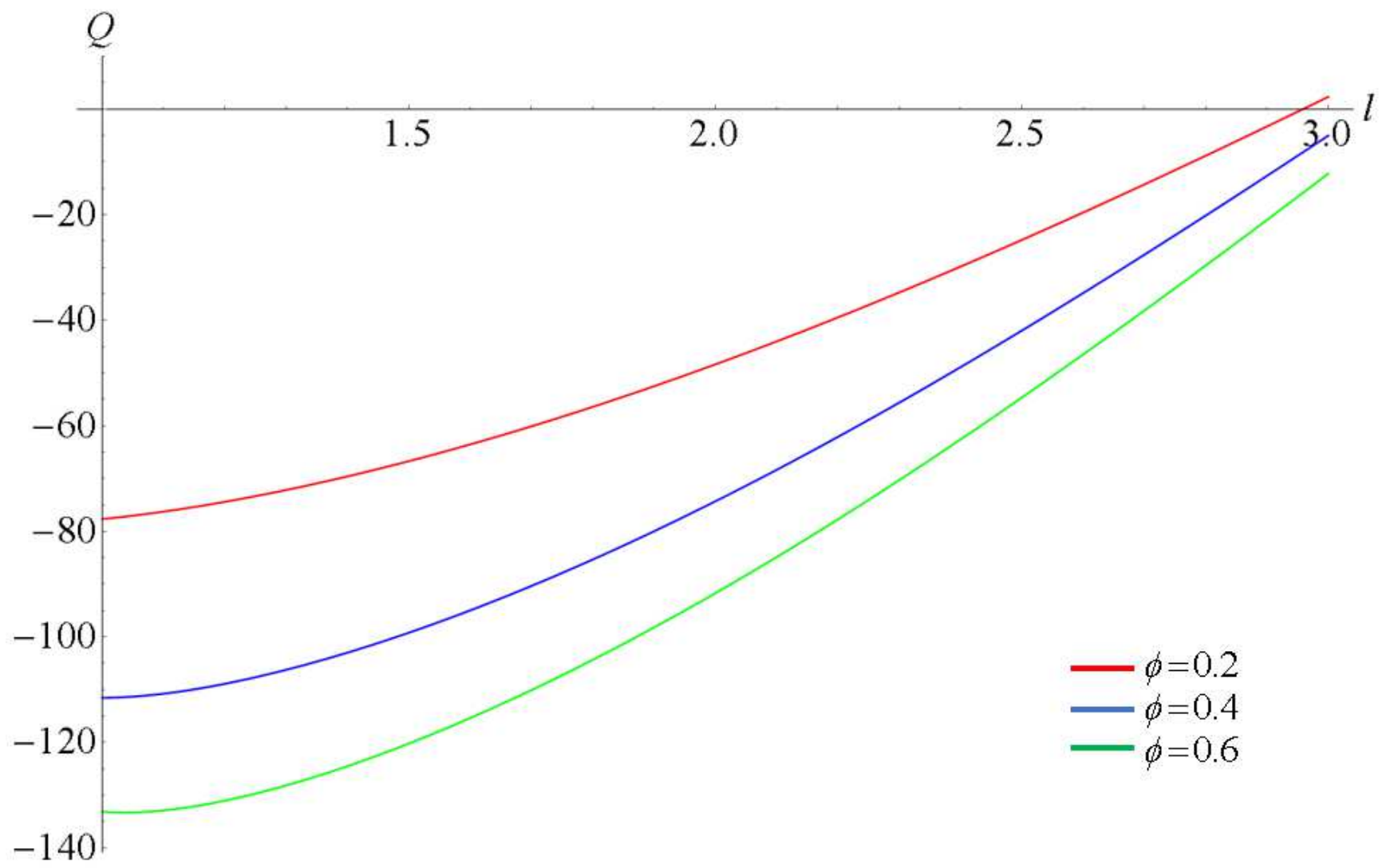

Fig. 3: Variation of flow rate with porosity $\phi$.

\subsubsection{Effect of viscosity ratio $\lambda$}

The effects of viscosity ratio parameter $(\lambda=0.05,0.1,1)$ on volumetric flow rate are represented by figure 4 with some fixed value of micropolar parameter $M=0.2$, porosity $\phi=0.5$, Hartmann number $\left(H_{1}=1.5\right)$, Darcy numbers $\left(k_{1}=0.3, k_{2}=4, k_{3}=5\right)$ and conductivity ratio parameters $\left(\Lambda_{2}=0.5, \Lambda_{3}=0.25\right)$. Within the porous cylindrical shell 
containing Newtonian fluid, the graph of flow rate increases as viscosity ratio parameter increases and for outer porous cylindrical shell region through which micropolar fluid is flowing, it decays as the parameter $\lambda$ increases.

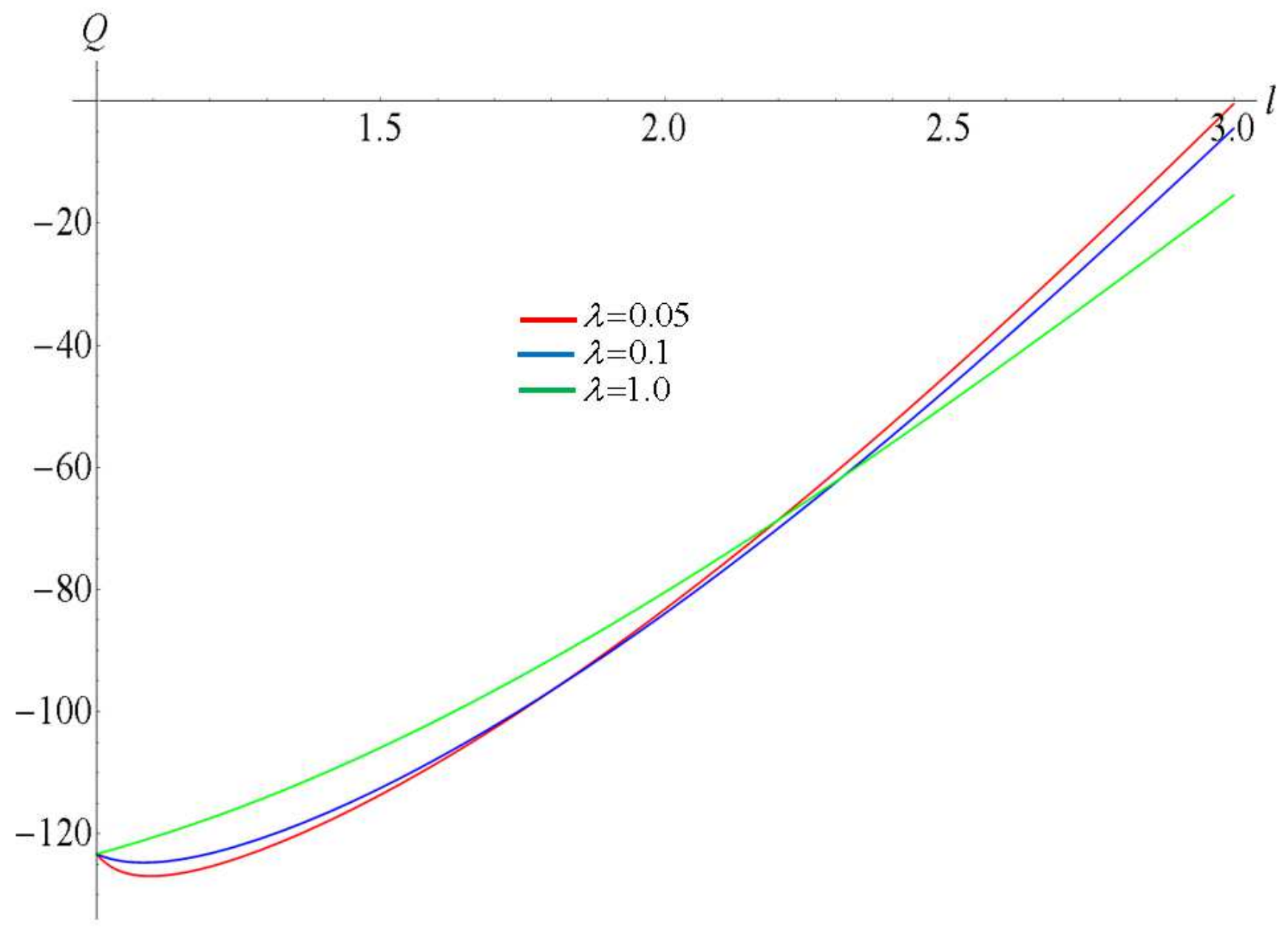

Fig. 4: Variation of flow rate with viscosity ratio parameter $\lambda$.

\subsubsection{Effect of Hartmann number $H_{1}$}

For the various values of magnetic parameter i.e. Hartmann number $\left(H_{1}=1,1.25,1.5\right)$, the graphs of the flow rate are plotted, and shown in figure 5 with $M=0.2, \phi=0.5, \lambda=$ $0.2, k_{1}=0.3, k_{2}=4, k_{3}=5$ and conductivity ratio parameters $\Lambda_{2}=0.5, \Lambda_{3}=0.25$. For parameter $1<l<2.75$, flow rate increases as Hartmann number increases and further, flow rate decreases as the magnetic parameter $H_{1}$ increases whenever $l>2.75$. 


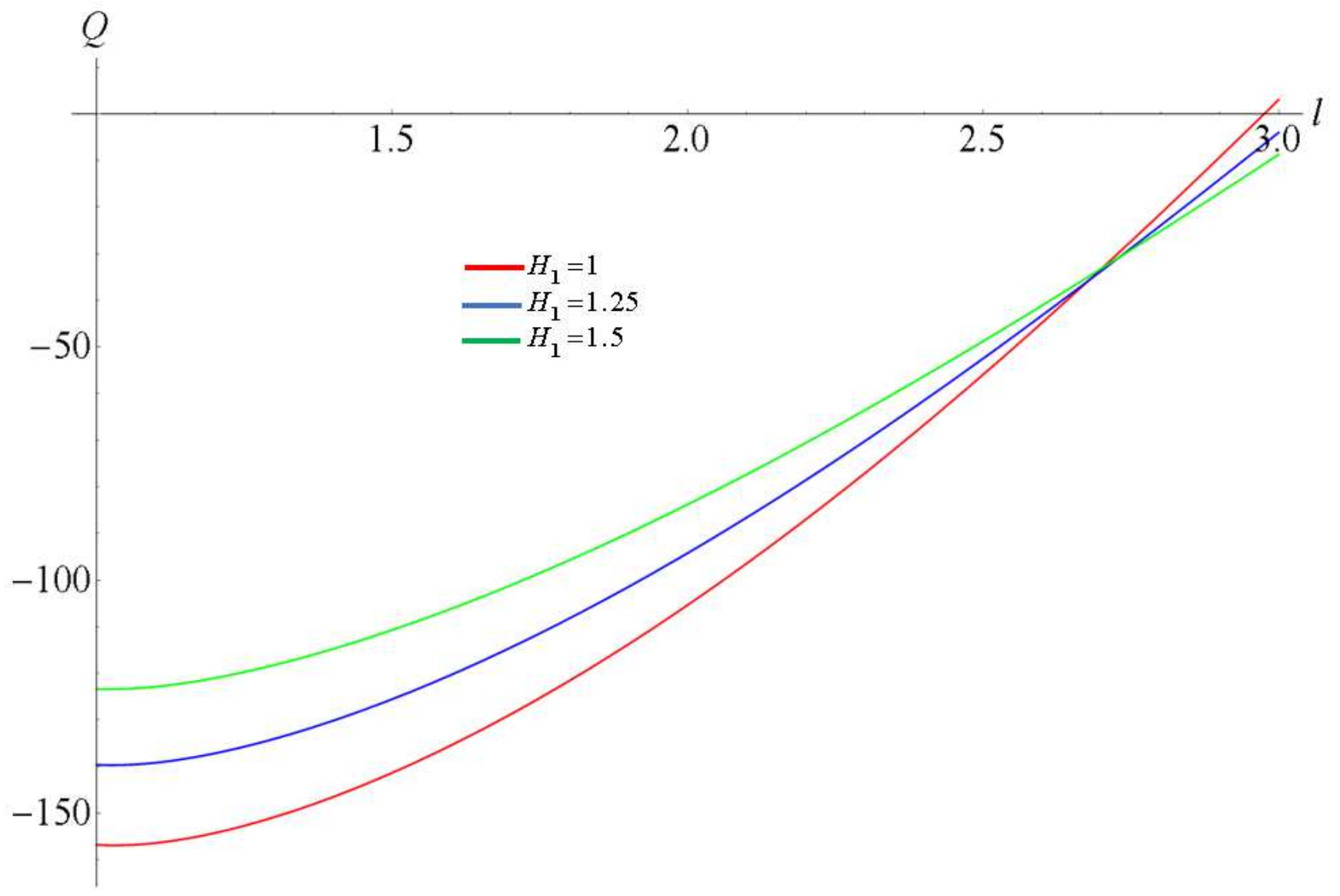

Fig. 5: Variation of flow rate with Hartmann number $H_{1}$.

\subsubsection{Effect of conductivity ratio parameter $\Lambda_{2}$}

Figure 6 represents the variation of the flow rate for the various values of conductivity ratio parameter $\left(\Lambda_{2}=0.2,1,2\right)$. Graphical behaviors are plotted with the some fixed value of flow parameters as flow parameters $M=0.2, \phi=0.5, \lambda=0.2, k_{1}=0.3, k_{2}=4, k_{3}=5$ and along with conductivity ratio parameter $\Lambda_{3}=0.25$. The obtained graph is similar to graph which is plotted for case of viscosity ratio parameter. We observed that for parameter $1<l<2.25$, flow rate increases as parameter $\Lambda_{2}$ increases and further, flow rate decreases as the conductivity ratio parameter $\Lambda_{2}$ increases whenever $l>2.25$. 


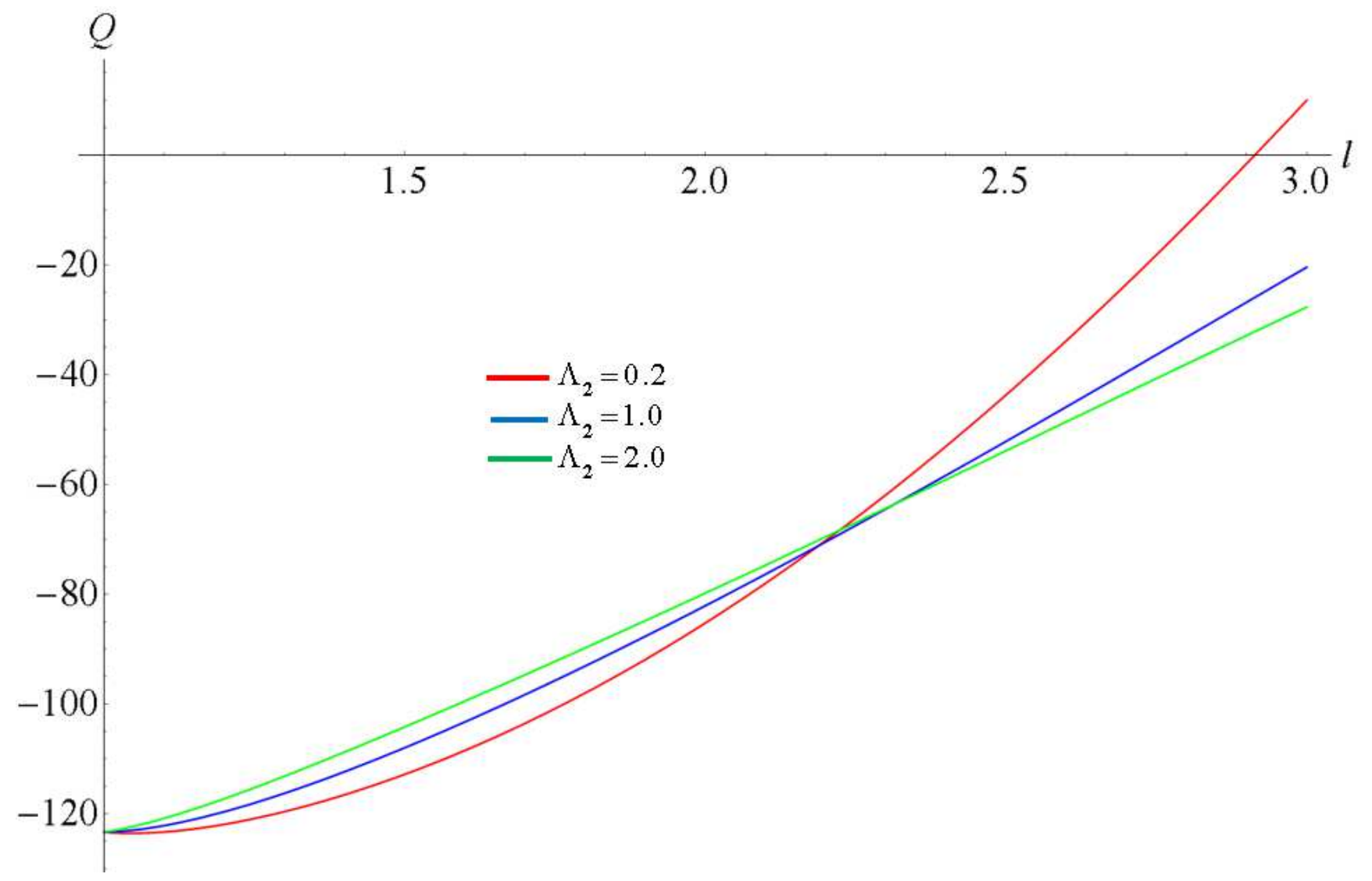

Fig. 6: Variation of flow rate with conductivty ratio parameter $\Lambda_{2}$.

\subsubsection{Effect of conductivity ratio parameter $\Lambda_{3}$}

Variation of the flow rate versus parameter $l$ is plotted for different values of flow parameter $\left(\Lambda_{3}=0.2,1,2\right)$. To draw the graph, we have considered some fixed value of flow parameters as micropolar parameter $(M=0.2)$, porosity $(\phi=0.5)$, viscosity ratio $(\lambda=0.2)$, Hartmann number $\left(H_{1}=1.5\right)$, Darcy numbers $\left(k_{1}=0.3, k_{2}=4, k_{3}=5\right)$ and conductivity ratio parameter $\left(\Lambda_{2}=0.25\right)$. After investigation, we find that the obtained graph varies smoothly for the case of conductivity ratio parameter $\left(\Lambda_{3}\right)$. It is observed that the volumetric flow rate increases as the ratio parameter $\Lambda_{3}$ increases (Figure 7 ). Also, these graphs converge with variation of the parameter $l$. 


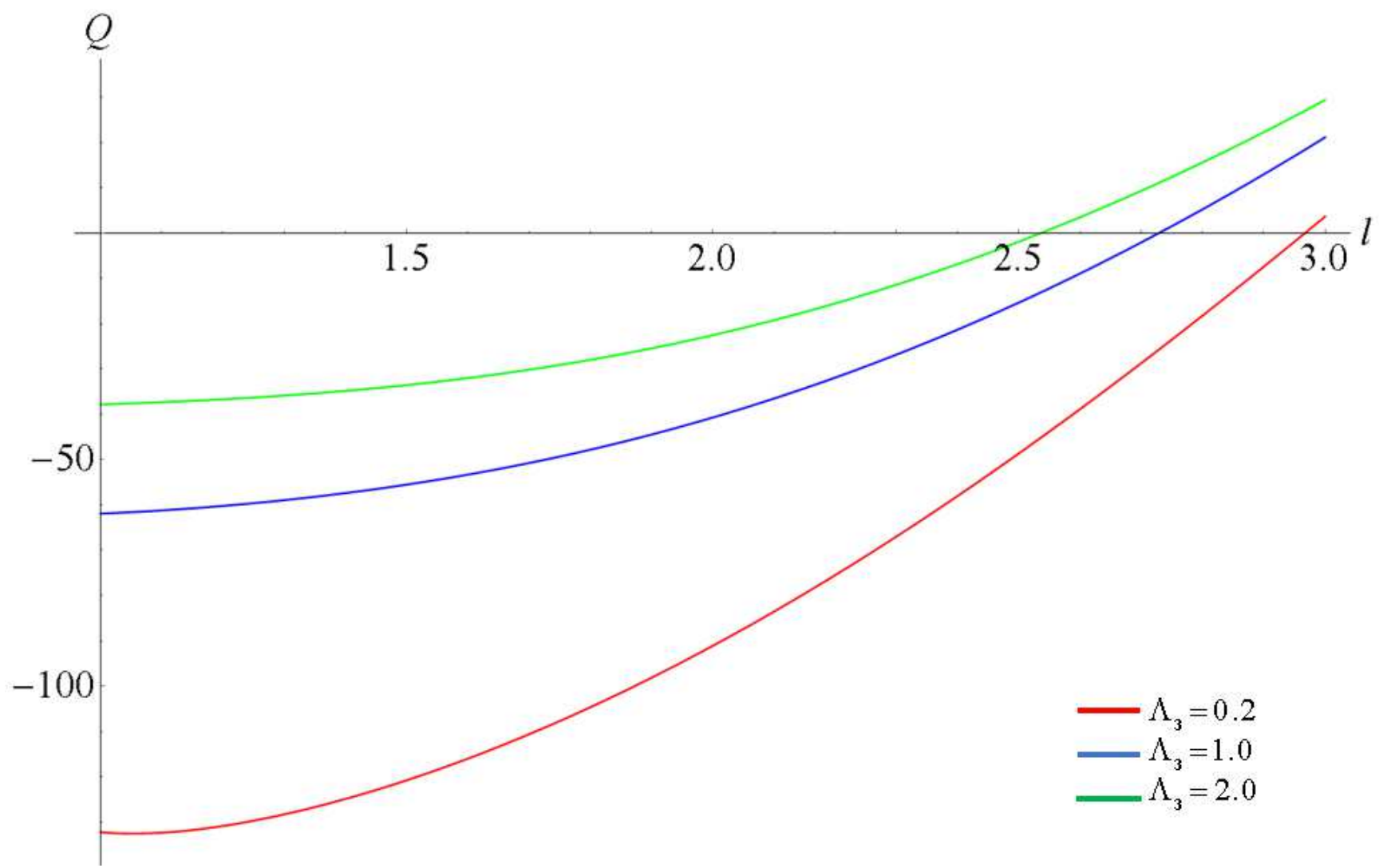

Fig. 7: Variation of flow rate with conductivty ratio parameter $\Lambda_{3}$.

\subsubsection{Effect of Darcy number $k_{1}$}

With consideration of the fixed value of flow parameters as $M=0.5, \quad \phi=0.3, \lambda=0.5$, $H_{1}=0.5, k_{2}=0.4, k_{3}=1, \Lambda_{2}=1, \Lambda_{3}=1$, volumetric flow rate is plotted for different values of Darcy numbers $\left(k_{1}=0.01,0.1,1\right)$. It is observed that the volumetric flow rate decreases as the Darcy number $k_{1}$ increases and varies continuosly along with variation of parameter $l$ (Figure 8). Also, these graphs intersect to each other in some neighourhood of parameter $l=2.5$. 


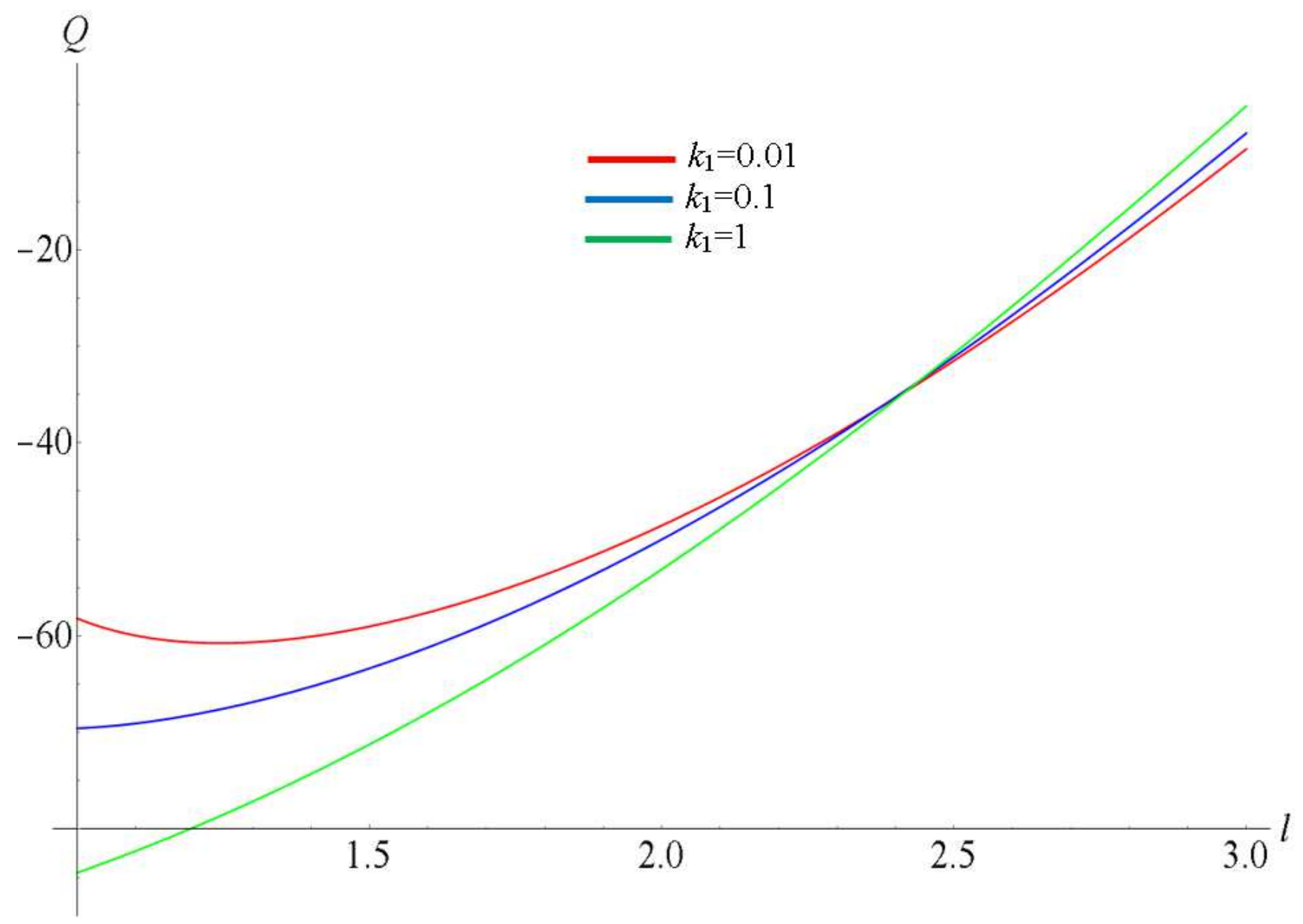

Fig. 8: Variation of flow rate with Darcy number $k_{1}$.

\subsubsection{Effect of Darcy number $k_{2}$}

Figure 9 represents the graphical illustration of flow rate with flow parameters as $M=0.2$, $\phi=0.5, \lambda=0.2, H_{1}=1.5, k_{1}=4, k_{3}=5, \Lambda_{2}=0.5, \quad \Lambda_{3}=0.25$, for the various values of Darcy number $\left(k_{2}=0.1,0.2,0.6\right)$. Investigation offers that the volumetric flow rate is of dual nature firstly it decreases as the Darcy number $k_{1}$ increases whenever $1<l<2$. Also, these graphs intersect to each other in some neighbourhood of parameter $l=1.75$, and latter graph of flow rate diverges continuously along with variation of parameter $l$ whenever $l>2$. 


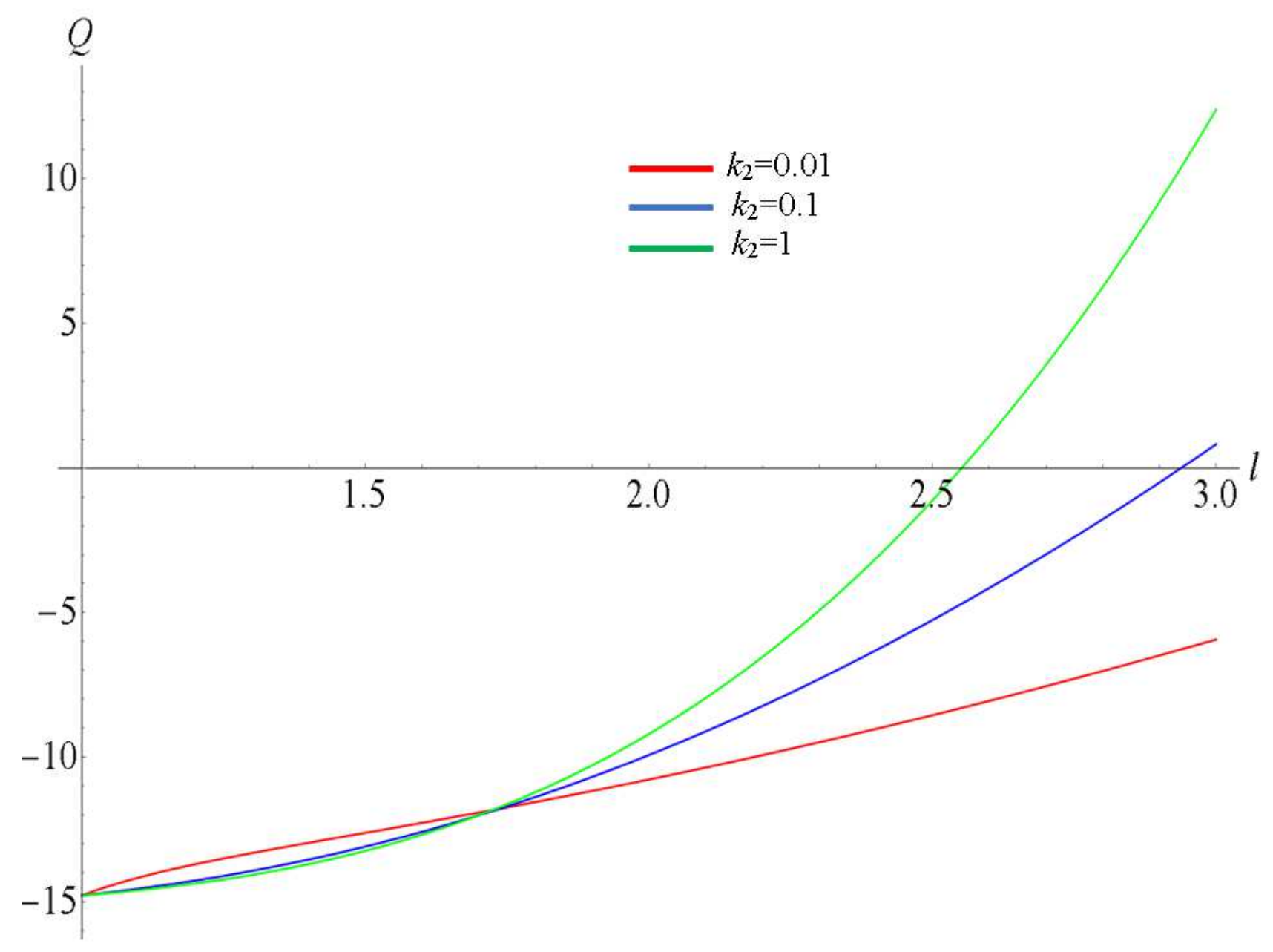

Fig. 9: Variation of flow rate with Darcy number $k_{2}$.

\subsubsection{Effect of Darcy number $k_{3}$}

With consideration of fixed value of flow parameters as $M=0.2, \quad \phi=0.5, \quad \lambda=0.2$, $H_{1}=1.5, k_{1}=5, k_{2}=4, \Lambda_{2}=0.5, \quad \Lambda_{3}=0.25$, volumetric flow rate is plotted for different values of Darcy numbers $\left(k_{3}=0.01,0.1,1\right)$. It is observed that the volumetric flow rate decreases rapidly as the Darcy number $k_{3}$ increases. Also, the graphs decrease for large value of Darcy number and converge for large values of parameter $l$ (Figure 10). 


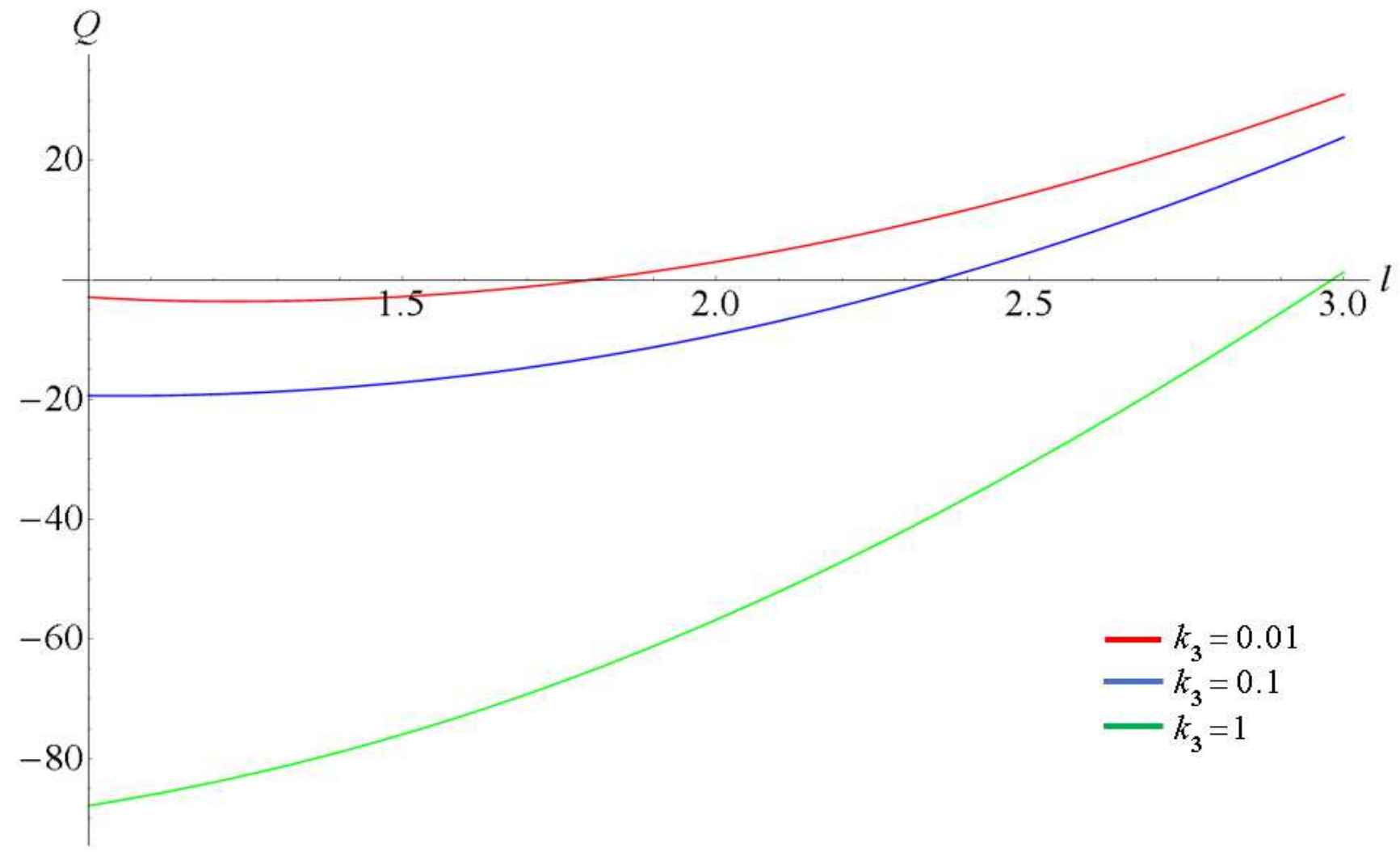

Fig. 10: Variation of flow rate with Darcy number $k_{3}$.

\subsection{Discussion on micropolar fluid velocity $(v)$}

With the variation of radial coordinate $0 \leq r \leq 5$, the micropolar fluid velocity $v$ is plotted and explained its graphical natures for various values of fluid flow parameters. Flow parameters are as micropolar parameter, porosity, viscosity ratio, Hartmann number, conductivity ratio parameters, and Darcy numbers, represented by mathematical symbols $M, \phi, \lambda, H_{1}, \Lambda_{2}, \Lambda_{3}, k_{1}, k_{2}, k_{3}$, respectively. In every case, couple stress parameter $L=2$, pressure gradient $P=-3$, length ratio parameter $l=3, m=5$ are used and cylindrical regions $(0 \leq r \leq 1),(3 \leq r \leq 5)$ are containing the micropolar fluids of different permeabilities while Newtonian fluid is passing through the region $(1 \leq r \leq 3)$.

\subsubsection{Effect of micropolar parameter (M)}

Variation of micropolar fluid velocity is plotted for different values of micropolar parameter $(M=0.1,0.4,0.5)$ by considering the values of some flow parameters as 
$\lambda=0.2, \quad k_{1}=0.3, \quad k_{2}=4, k_{3}=5, \phi=0.5, H_{1}=1.5, \Lambda_{2}=0.5, \Lambda_{3}=0.25$.

Within the fluid region $(0 \leq r \leq 1)$, fluid velocity increases as flow parameter $M$ varies and fluid flowing through Newtonian region $(1 \leq r \leq 3)$, velocity decreases as micropolar parameter increases. Newtonian fluid particles move along a parabolic path within region $(1<r<3)$ whose vertex is upwards (Fig. 11). Also, the path travelled by fluid particles in outer cylindrical region is cycloidal and fluid velocity $v$ decreases as parameter $m$ increases with variation of radial coordinate $r$.

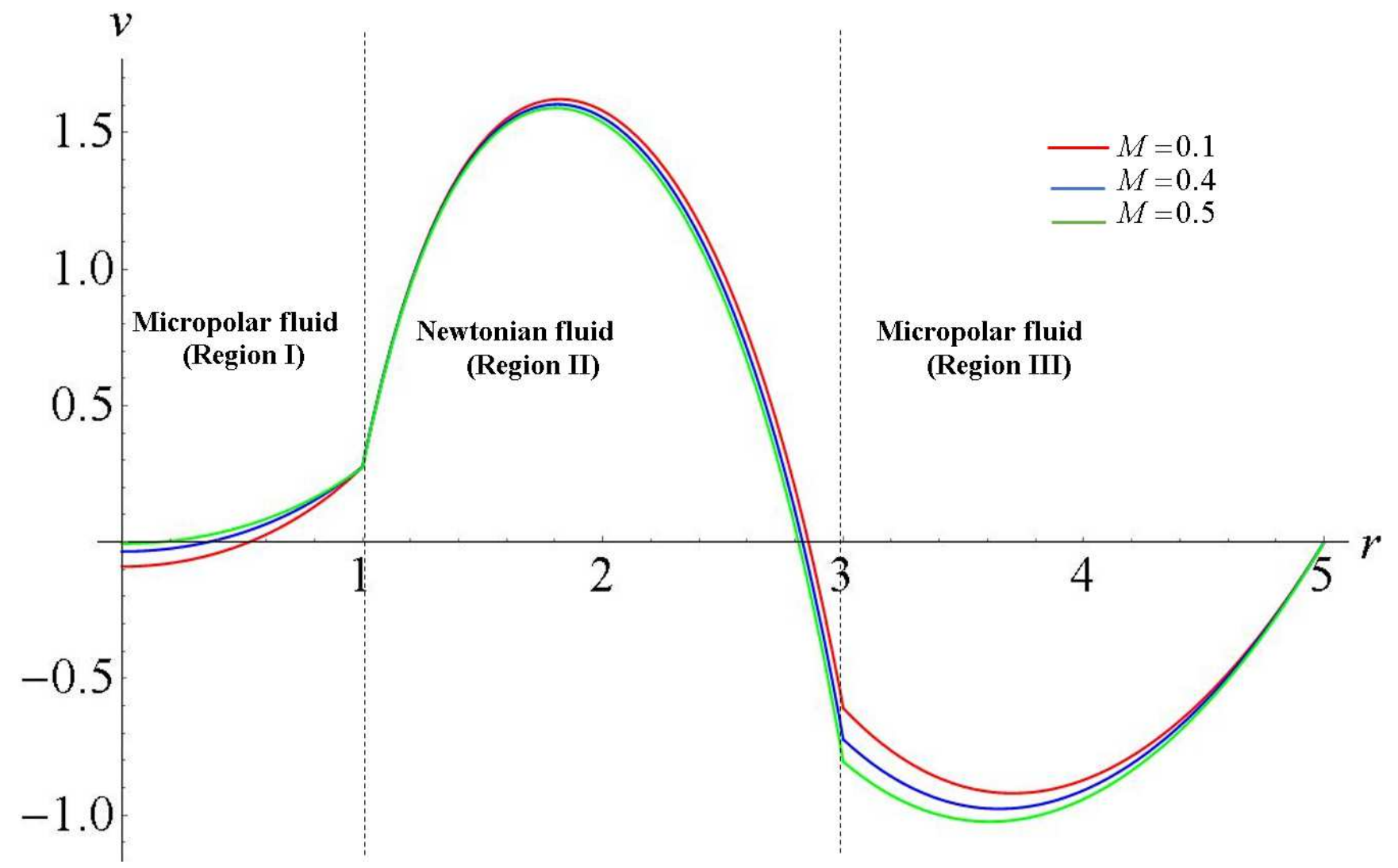

Fig. 11: Variation of fluid velocity with micropolar parameter $M$.

\subsubsection{Effect of porosity $(\phi)$}

Figure 12 shows the variation of fluid velocity for flow parameter $(\phi=0.2,0.3,0.4)$ by considering the values of some flow parameters as $M=0.2, \lambda=0.2, k_{1}=0.3, k_{2}=$ $4, k_{3}=5, H_{1}=1.5, \Lambda_{2}=0.5, \Lambda_{3}=0.25$. Within the fluid regions $(0 \leq r \leq 1)$ and $(3 \leq$ $r \leq 5)$ which are filled with micropolar fluid flows, the micropolar fluid velocity decreases as porosity increases and also, the flow through Newtonian fluid region $(1 \leq r \leq 3)$, velocity increases as parameter $\phi$ increases. Fluid particles move along a parabolic path 
within region $(1<r<3)$. With variation of radial coordinate curve $r$, streamlines drawn by fluid particles are parabolic.

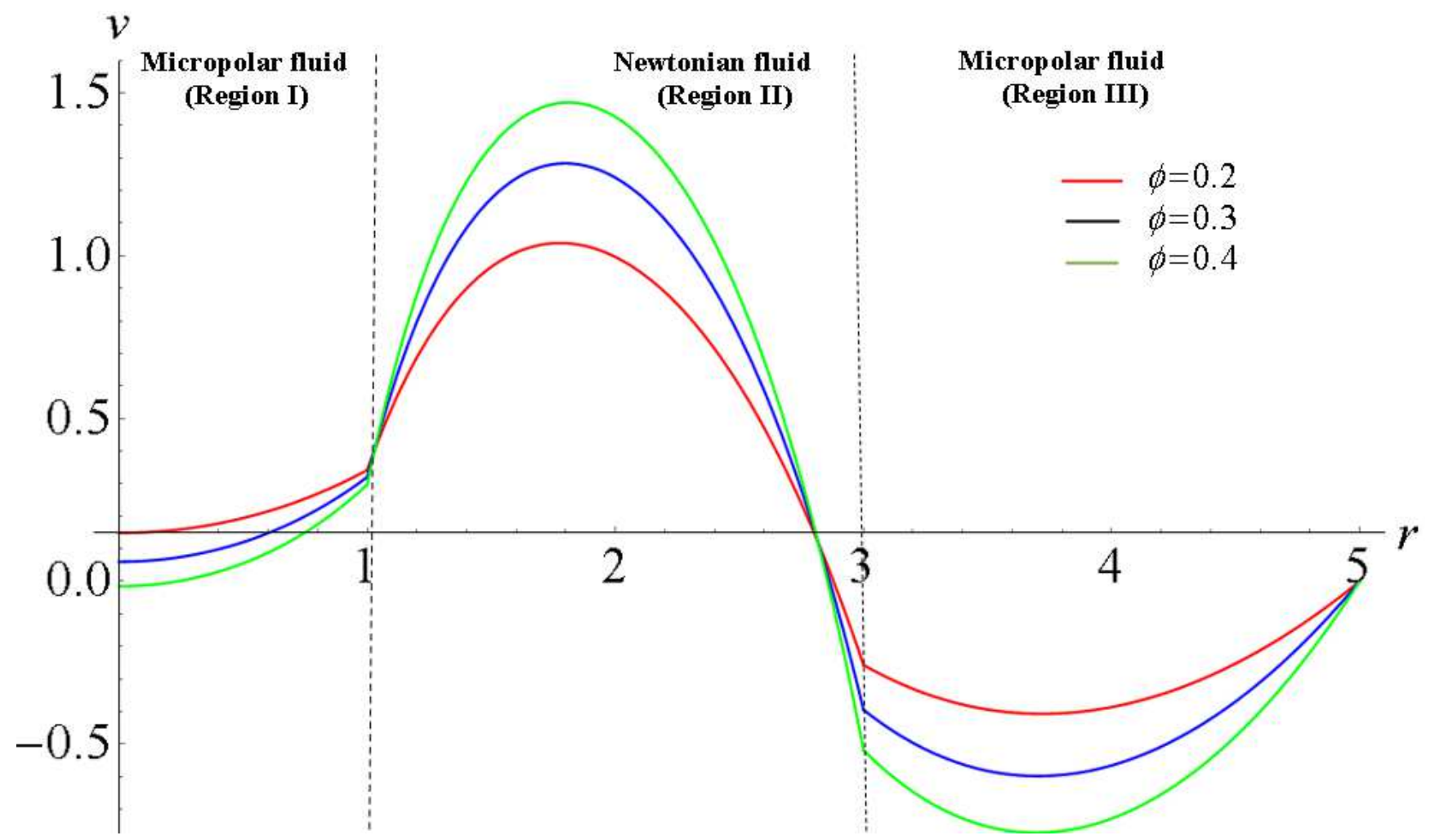

Fig. 12: Variation of fluid velocity with porosity $\phi$.

\subsubsection{Effect of viscosity ratio $(\lambda)$}

Variation of fluid velocity is plotted in the figure (13) for viscosity ratio $(\lambda=1.0,1.5,2.0)$ along with some fixed values of fluid flow parameters as $M=0.2, \phi=0.5, k_{1}=3, k_{2}=$ $4, k_{3}=5, H_{1}=1.5, \Lambda_{2}=0.5, \Lambda_{3}=0.25$. The fluid velocity decreases as flow parameter $\lambda$ increases in the fluid regions $(0 \leq r<3)$, and fluid flowing through outer cylindrical region $(3 \leq r \leq 5)$, velocity increases as viscosity ratio parameter increases. The path traced by fluid particles through cylindrical region is parabolic. 


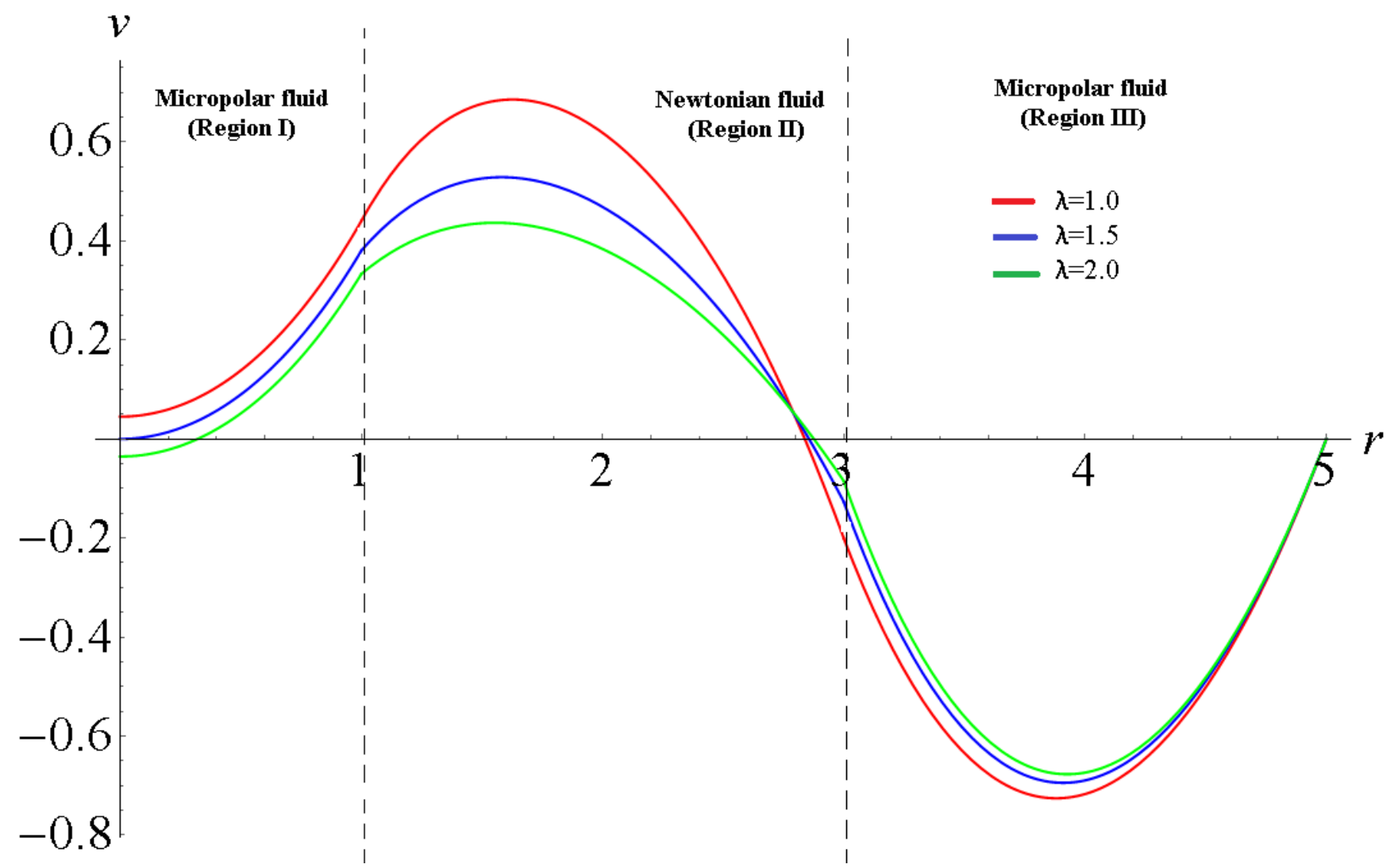

Fig. 13: Variation of fluid velocity with viscosity ratio $\lambda$.

\subsubsection{Effect of Hartmann number $\left(H_{1}\right)$}

Plot of fluid velocity for Hartmann number $\left(H_{1}=0.1,0.5,1.0\right)$ is reported in figure (14) by considering the values of fluid flow parameters as $M=0.2, \lambda=0.2, k_{1}=0.3, k_{2}=$ $4, k_{3}=5, \phi=0.5, \Lambda_{2}=0.5, \Lambda_{3}=0.25$. Within the fluid region $(0 \leq r \leq 5)$, fluid velocity decreases whenever the flow parameter i.e. Hartmann number $H_{1}$ increases. 


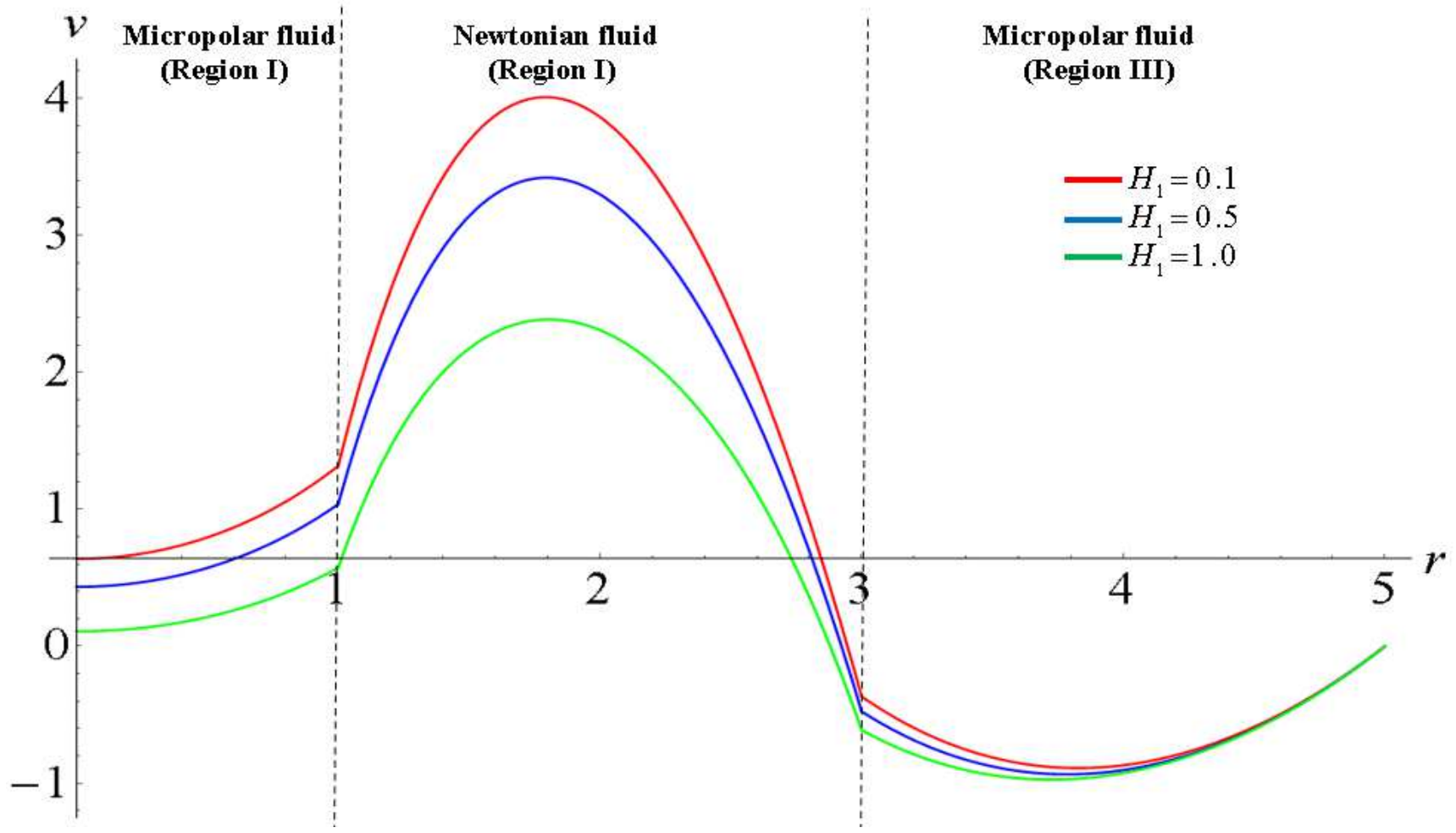

Fig. 14: Variation of fluid velocity with Hartmann number $H_{1}$.

\subsubsection{Effect of conductivity ratio $\left(\Lambda_{2}\right)$}

Figure 15 shows the variation of fluid velocity for various values of conductivity ratio $\left(\Lambda_{2}=0.1,0.3,0.5\right)$ by considering the some fixed values of fluid flow parameters as $M=0.2, \lambda=0.2, \quad k_{1}=0.3, k_{2}=4, k_{3}=5, \phi=0.5, H_{1}=1.5, \Lambda_{3}=0.25$. Graph for the conductivity ratio $\Lambda_{2}$ is similar to graph plotted for case of Hartmann number. For the fluid region $(0 \leq r \leq 5)$, fluid velocity decreases as flow parameter $\Lambda_{2}$ varies and path traced by each fluid particles is a parabolic path. 


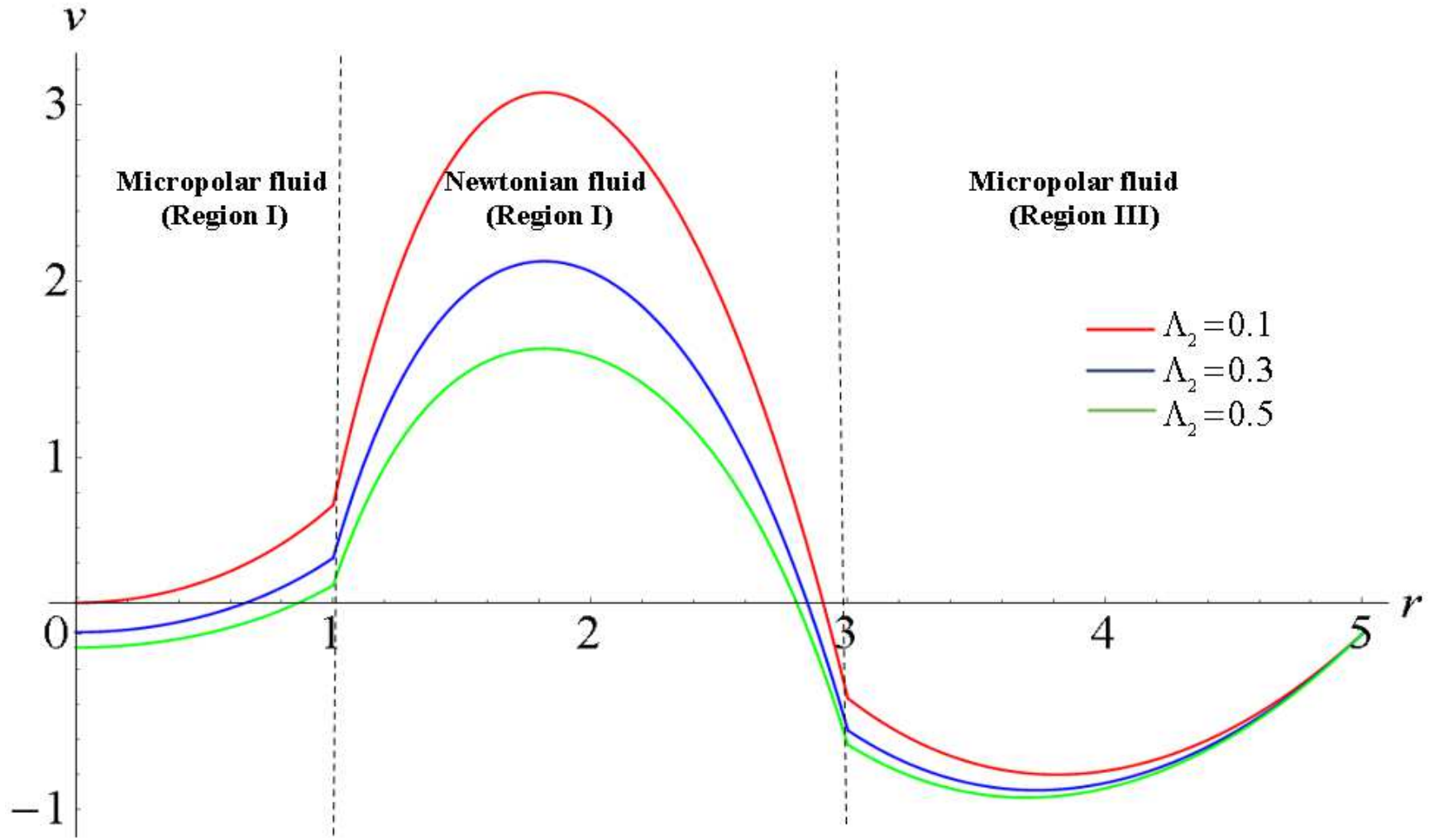

Fig. 15: Variation of fluid velocity with conductivity ratio parameter $\Lambda_{2}$.

\subsubsection{Effect of conductivity ratio $\left(\Lambda_{3}\right)$}

Variation of fluid velocity is represented for conductivity ratio $\left(\Lambda_{3}=0.2,0.5,0.8\right)$ by considering the values of fluid flow parameters as $\lambda=0.2, k_{1}=0.3, k_{2}=4, k_{3}=5, \phi=$ $0.5, H_{1}=1.5, M=0.2, \Lambda_{2}=0.25$. Within the region $(0 \leq r \leq 5)$, fluid velocity increases as flow parameter $\Lambda_{3}$ increases (Fig. 16). Streamlines, i.e. path traced out by each fluid particle in porous cylindrical regions is a parabolic curve along with variation of radial coordinate curve $r$. 


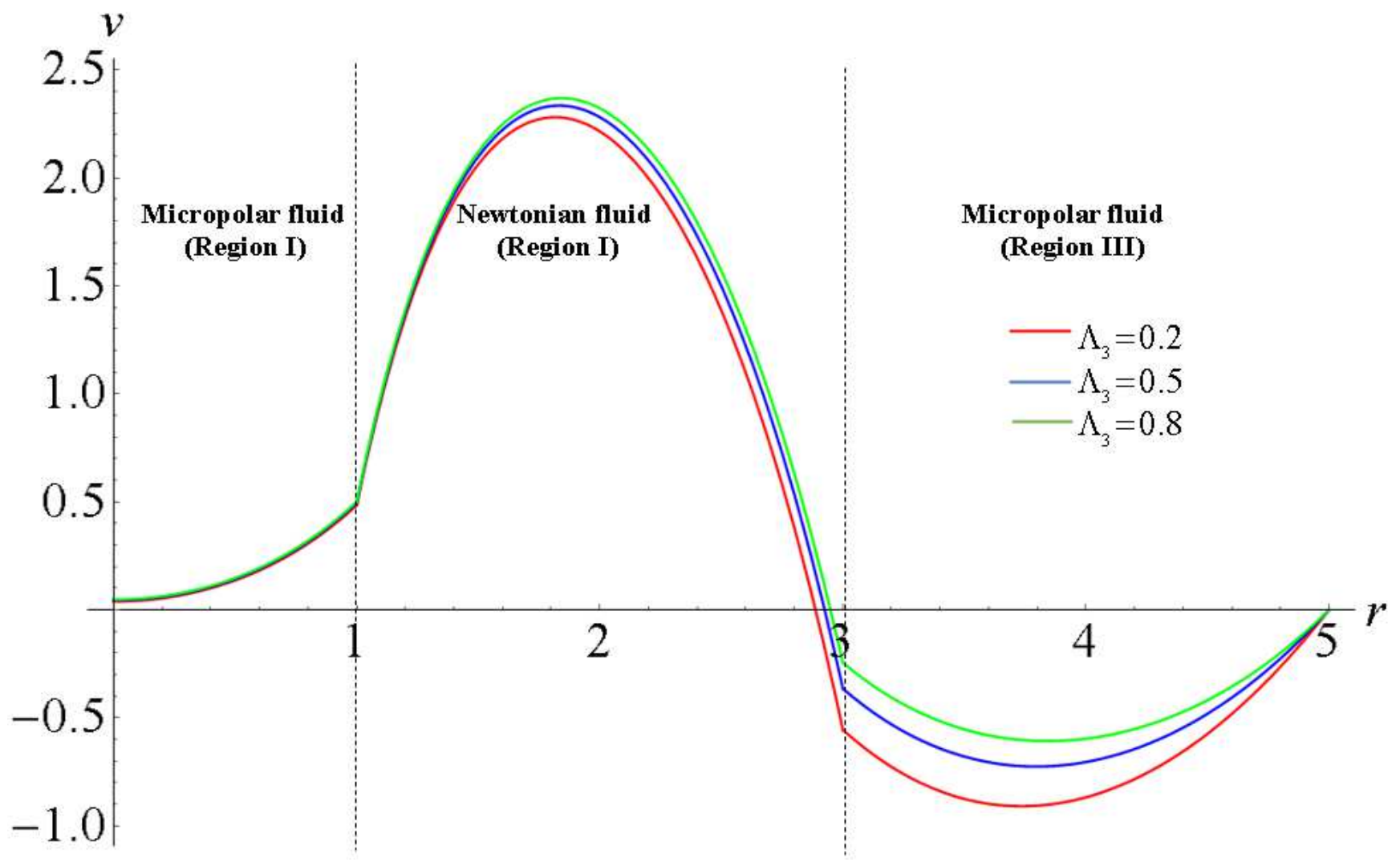

Fig. 16: Variation of fluid velocity with conductivity ratio parameter $\Lambda_{3}$.

\subsubsection{Effect of Darcy number $\left(k_{1}\right)$}

Graphical behavior of fluid velocity is plotted for Darcy number $\left(k_{1}=0.1,1.0,1.5\right)$ along with the fixed values of fluid flow parameters as $M=0.3, \lambda=0.2, k_{2}=4, k_{3}=5, \phi=$ 0.5, $H_{1}=0.5, \Lambda_{2}=0.5, \Lambda_{3}=0.25$ (Fig. 17). On the investigation, we get that the fluid velocity increases continuously as Darcy number $k_{1}$ increases with radial coordinate $r$. Variations in the fluid velocity are found more sharply in inner cylindrical regions $(0 \leq r \leq 3)$ compared to outer cylindrical region $(3 \leq r \leq 5)$. Streamlines coincide for each values of Darcy number through outer porous cylindrical region. 


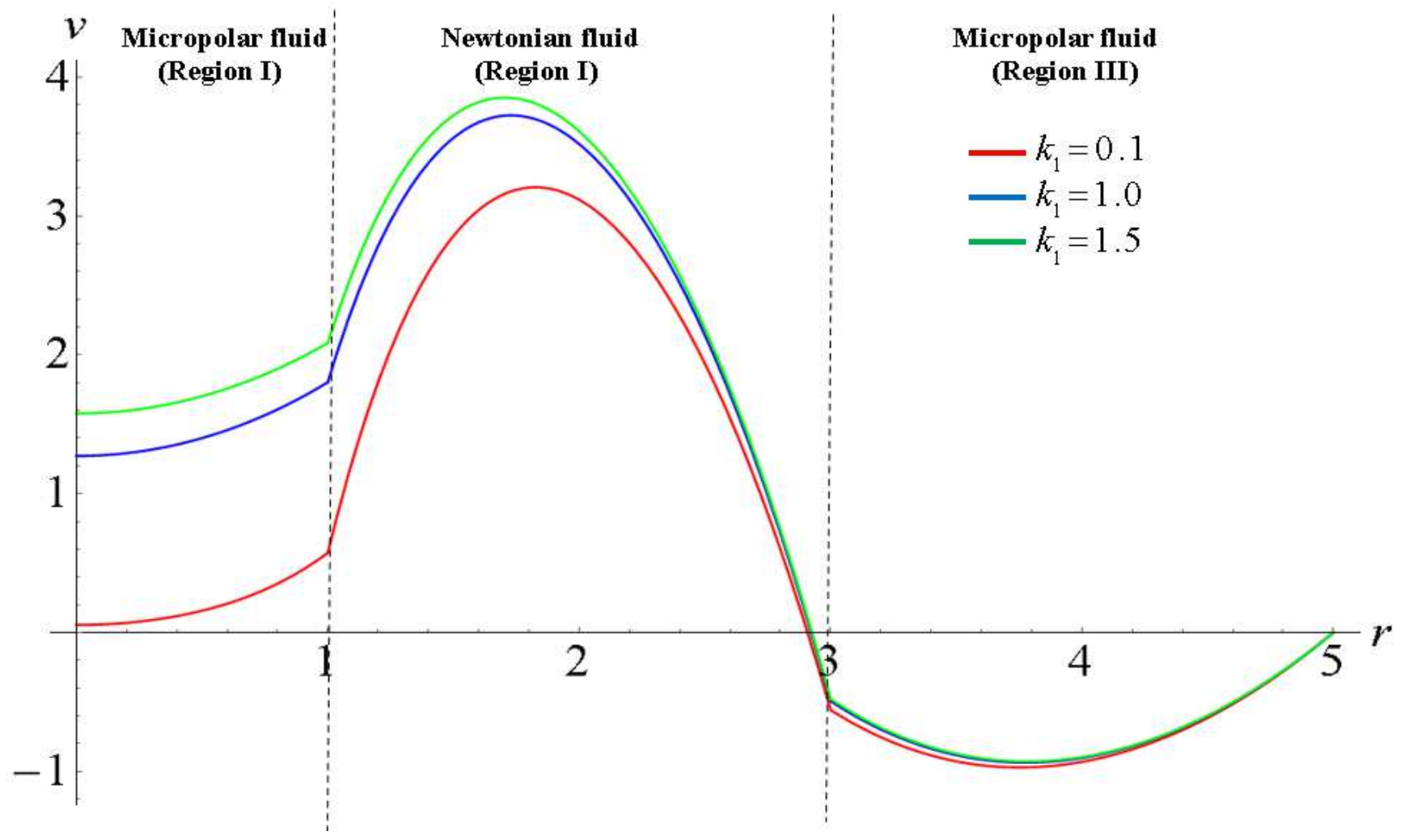

Fig. 17: Variation of fluid velocity with Darcy number $k_{1}$.

\subsubsection{Effect of Darcy number $\left(k_{2}\right)$}

Variation of the fluid velocity is investigated for viscosity ratio $\left(k_{2}=0.2,0.4,0.6\right)$ in the figure 18 by fixing the values of the fluid flow parameters as $M=0.2, \lambda=0.2, k_{1}=$ $0.3, k_{3}=5, \phi=0.5, H_{1}=1.5, \Lambda_{2}=0.5, \Lambda_{3}=0.25$. Within the fluid region $(0 \leq r \leq 5)$, fluid velocity increases as flow parameter i.e. Darcy number $k_{2}$ increases. 


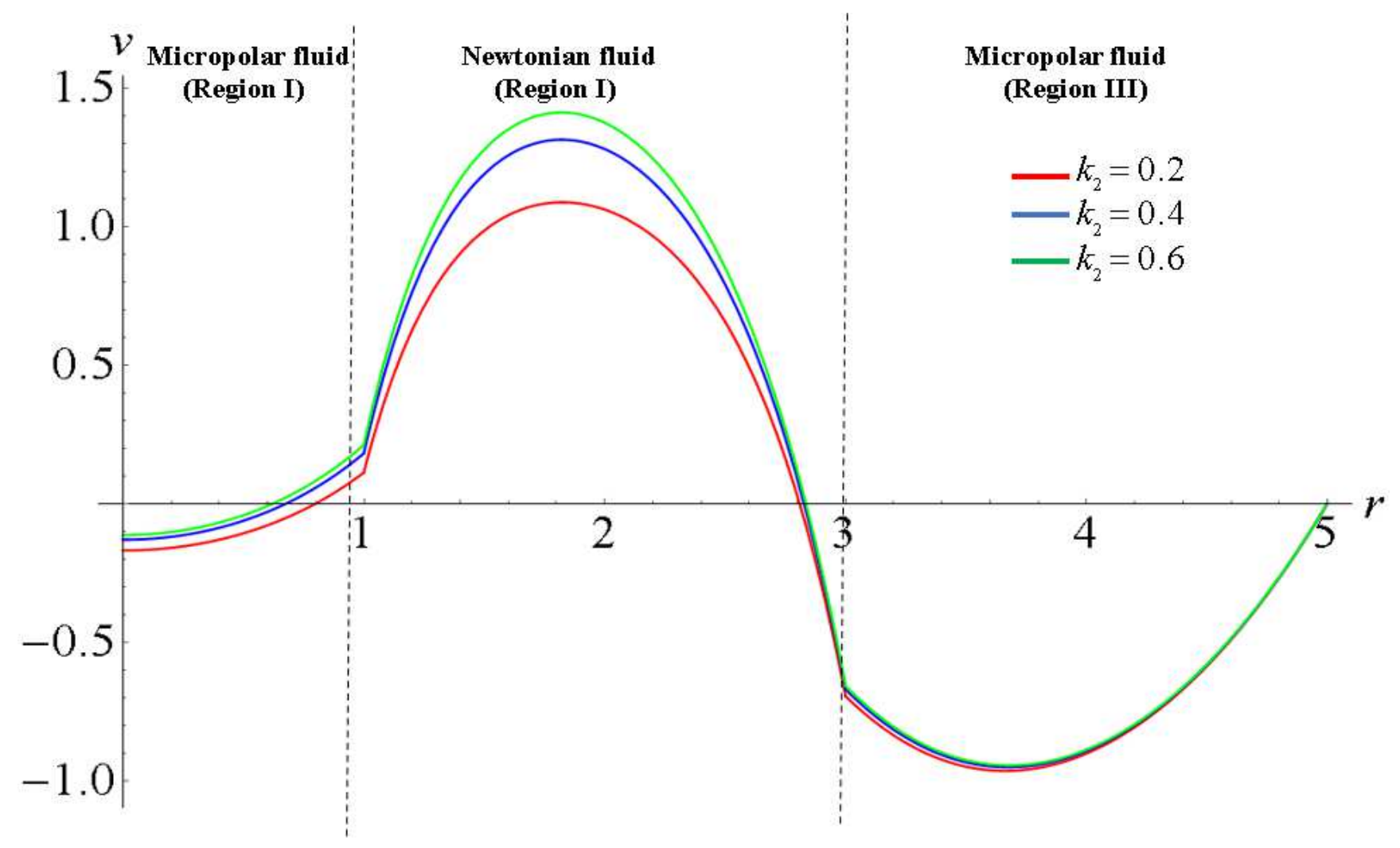

Fig. 18: Variation of fluid velocity with Darcy number $k_{2}$.

\subsubsection{Effect of Darcy number $\left(k_{3}\right)$}

Graph of fluid velocity is plotted for Darcy number $\left(k_{3}=0.2,0.4,0.6\right)$ corresponding to outer cylindrical region $(3<r<5)$. Considering the values of fluid flow parameters as micropolar parameter $(M=0.2)$ viscosity ratio $(\lambda=0.2)$, porosity $(\phi=0.5)$, Hartmann number $\left(H_{1}=1.5\right)$, conductivity ratio parameters $\left(\Lambda_{2}=0.5, \Lambda_{3}=0.25\right)$, Darcy numbers $\left(k_{1}=0.3, k_{2}=5\right)$ for plotting. Streamlines coincide for each values of Darcy number through inner porous cylindrical region $(0 \leq r \leq 1)$ only while different curves (i.e. streamlines) are obtained for remaining porous cylindrical regions $(1 \leq r \leq 5)$. Fluid velocity decreases as parameter $k_{3}$ increases and fluid particles move along a parabolic path within region $(1<r<3)$ whose vertex is upwards(Fig. 19). 


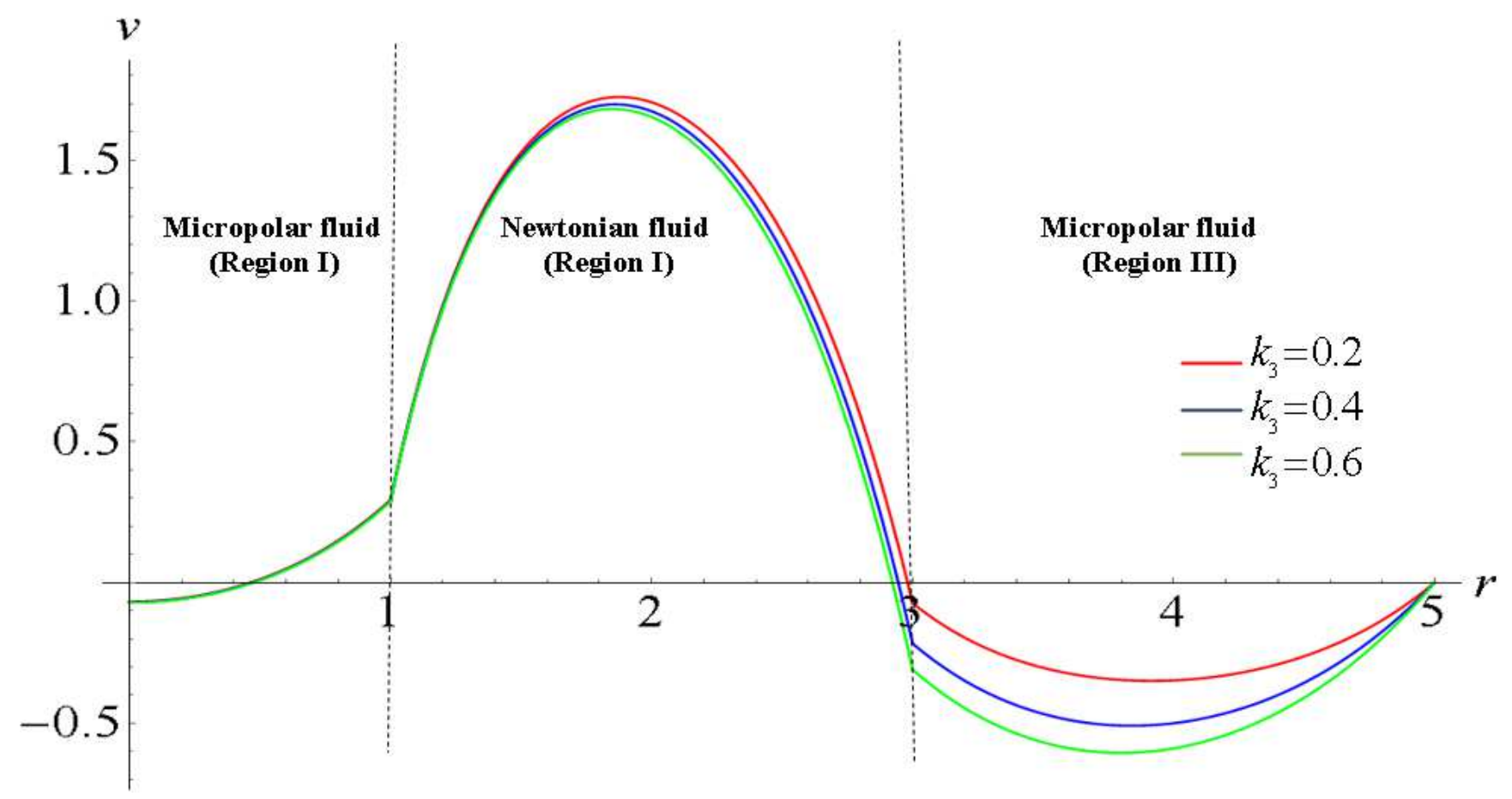

Fig. 19: Variation of fluid velocity with Darcy number $k_{3}$.

\section{Conclusion}

In the present study, we investigated the Newtonian fluid which is sandwiched between two immiscible micropolar fluids flowing through coaxial porous cylindrical regions under the influence of external uniform magnetic field. Motions of fluids are happening along the axis of cylinders and also, the direction of magnetic field is taken as the direction perpendicular to fluid motion. Fluid velocities (both Newtonian and micropolar), microrotations and shear stresses are determined using analyticity condition, continuity of velocity, continuity of microrotation and continuity of stresses. Volumetric flow rate is evaluated analytically. The graphical behaviors for flow rate and fluid velocity for various values of flow parameters (namely micropolar parameter, porosity, Hartmann number, viscosity ratio, Darcy numbers corresponding to each regions and conductivity ratio parameters) have been discussed. It is observed that the behaviour of volumetric flow rate is similar in the case of micropolar parameter, porosity, Darcy number for outer cylindrical region. Similar behaviours for velocity profiles are obtained in the case of Hartmann 
number and conductivity ratio parameter $\left(\Lambda_{2}\right)$. This research work will help and motivate young researchers to investigate the problems related to composite behaviours of Newtonian and non- Newtonian fluids. The significance of the proposed mathematical model is that it explains the effects of magnetic field, porosity, viscosity ratio, different permeabilities, i.e. Darcy numbers and conductivity ratios. Furthermore, this study will be useful in purification of contaminated groundwater, blood flows through veins/arteries, extraction of highly viscous crude oils, etc..

\section{References}

[1] Lukaszewicz, G (1999) Micropolar fluids: theory and applications. Springer, New York

[2] Eringen, AC (1966) Theory of micropolar fluids. J Math Mech 16:1-18

[3] Nowacki, W (1970) Theory of micropolar elasticity. Springer, New York

[4] Stokes, VK (1984) Theories of fluids with microstructure. Springer, New York

[5] Moosaie, A, Atefi, G (2008) Slow motion of a rotating circular cylinder through a micropolar fluid. Arch Mech 60:199-220

[6] Sherief, HH, Faltas, MS, Ashmawy, EA, Hameid, AMA (2014) Parallel and perpendicular flows of a micropolar fluid between slip cylinder and coaxial fictitious cylindrical shell in shell models. Eur Phys J Plus 129:217, DOI: 10.1140/epjp/i2014$14217-9$

[7] Khanukaeva, DY, Filippov, AN (2018) Isothermal flows of micropolar liquids: formulation of problems and analytical solutions. Colloid J 80:14-36

[8] Khanukaeva, DY, Filippov, AN, Yadav, PK, Tiwari, A (2019) Creeping flow of micropolar fluid parallel to the axis of cylindrical shells with porous layer. Eur J Mech/B Fluids 76:73-80 
[9] Yadav, PK, Jaiswal, S, Puchakatla, JY (2019) Micropolar fluid flow through the membrane composed of impermeable cylindrical particles coated by porous layer under the effect of magnetic field. Math Meth Appl Sci 43:1-13, DOI: https://doi.org/10.1002/mma.6016

[10] Yadav, PK, Verma, AK (2020), Analysis of immiscible Newtonian and nonNewtonian micropolar fluid flow through porous cylindrical pipe enclosing a cavity. Eur Phys J Plus 135:645, https://doi.org/10.1140/epjp/s13360-020-00672-6

[11] Deo, S, Maurya, DK, Filippov, AN (2020), Influence of magnetic field on micropolar fluid flow in a cylindrical tube enclosing an impermeable core coated with porous layer. Colloid J 82:649-660

[12] Yadav, PK, Jaiswal, S, Puchakatlaa, JY, Filippov, AN (2020) Poiseuille flow of micropolar-Newtonian fluid through concentric pipes filled with porous medium. Colloid J 82:333-341

[13] Maurya, DK, Deo, S (2020) Stream function solution of the Brinkman equation in parabolic cylindrical coordinates. Int J Appl Comput Math 6:167

[14] Nield, DA, Bejan, A (2006) Convection in porous media. Springer, New York

[15] Brinkman, HC (1947) A calculation of viscous force exerted by a flowing fluid on a dense swarm of particles. Appl Sci Res A1:27-34

[16] Kaviany, M (1985) Laminar flow through a porous channel bounded by isothermal parallel plates. Int J Heat Mass Transfer 28:851-858

[17] Tiwari, A, Deo, S (2013) Pulsatile flow in a cylindrical tube with porous walls: applications to blood flow. J Porous Media 16: 335-340

[18] Madasu, KP, Bucha, T (2019) Impact of magnetic field on flow past cylindrical shell using shell model. J Braz Soc Mech Sci Engg 41:320, https://doi.org/10.1007/s40430019-1820-x

[19] Deo, S, Maurya, DK (2019) Generalized stream function solution of the Brinkman equation in the cylindrical polar coordinates. Spec Top Rev Porous Med 10:421-428 
[20] Maurya, DK, Deo, S, Khanukaeva, DY (2020) Analysis of Stokes flow of micropolar fluid through a porous cylinder, Math Meth Appl Sci, DOI: 10.22541/au.159767833.38840439 
Figures

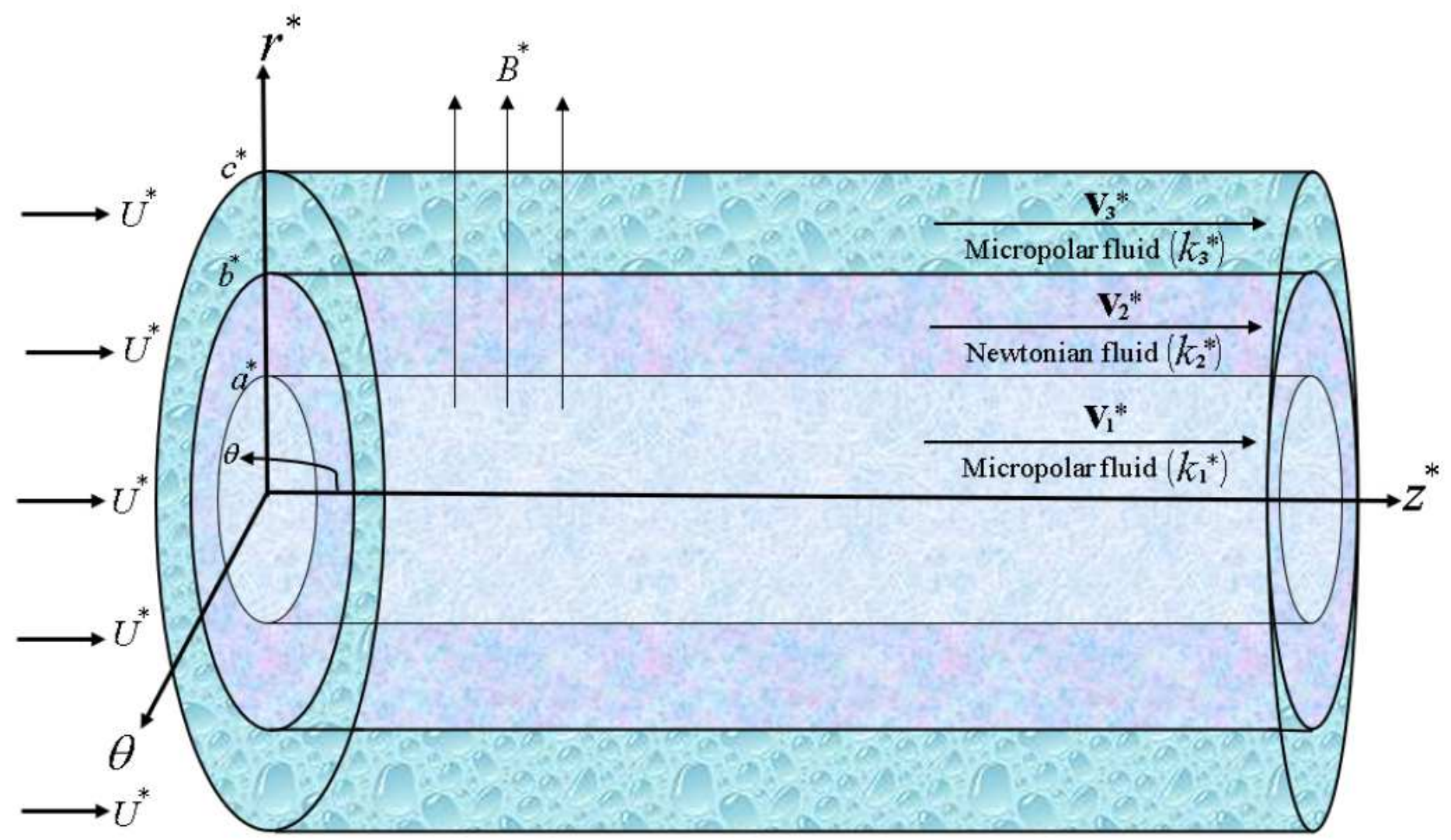

Figure 1

Schematic diagram of the problem 


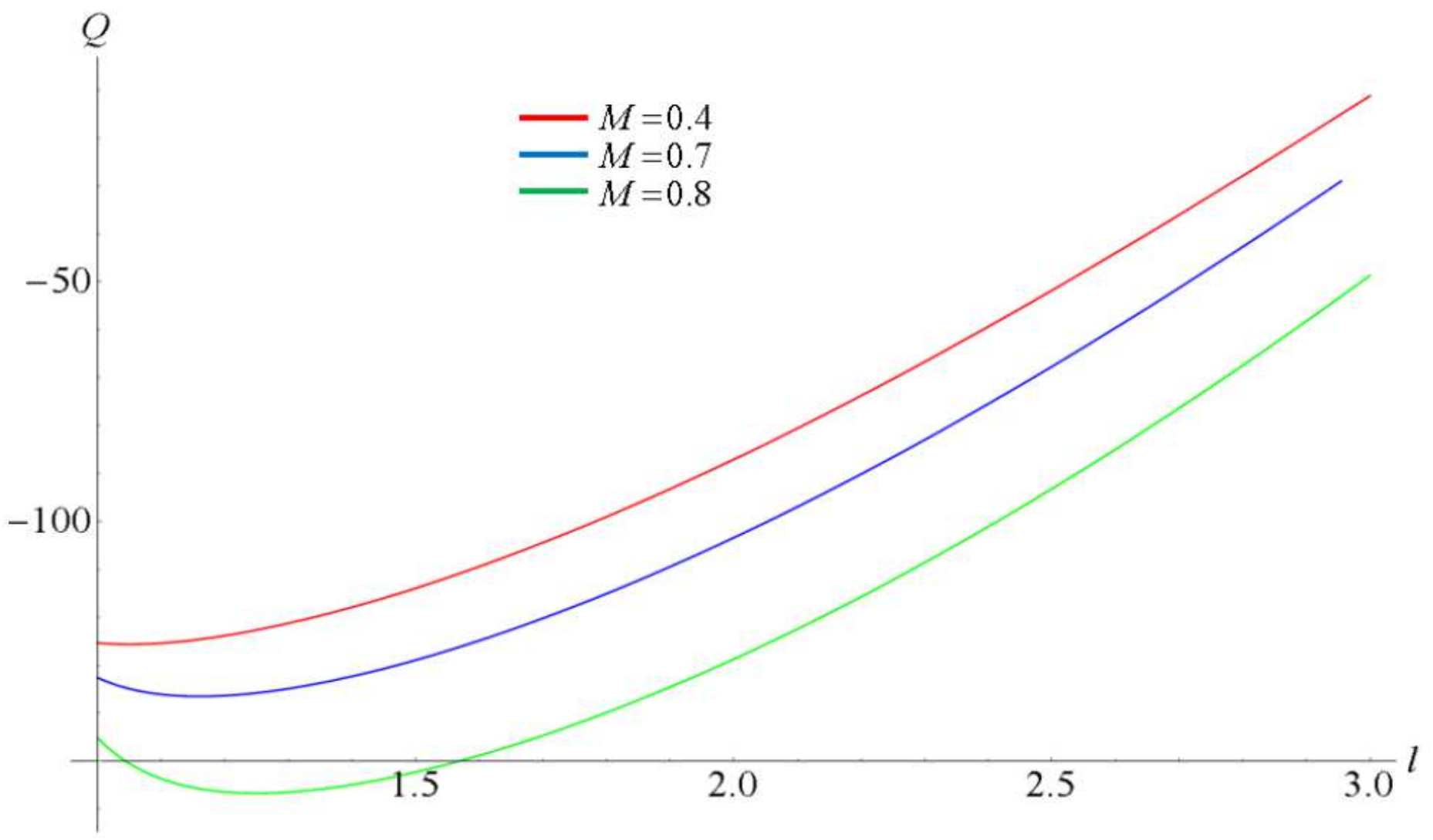

Figure 2

Variation of flow rate with micropolar parameter M. 


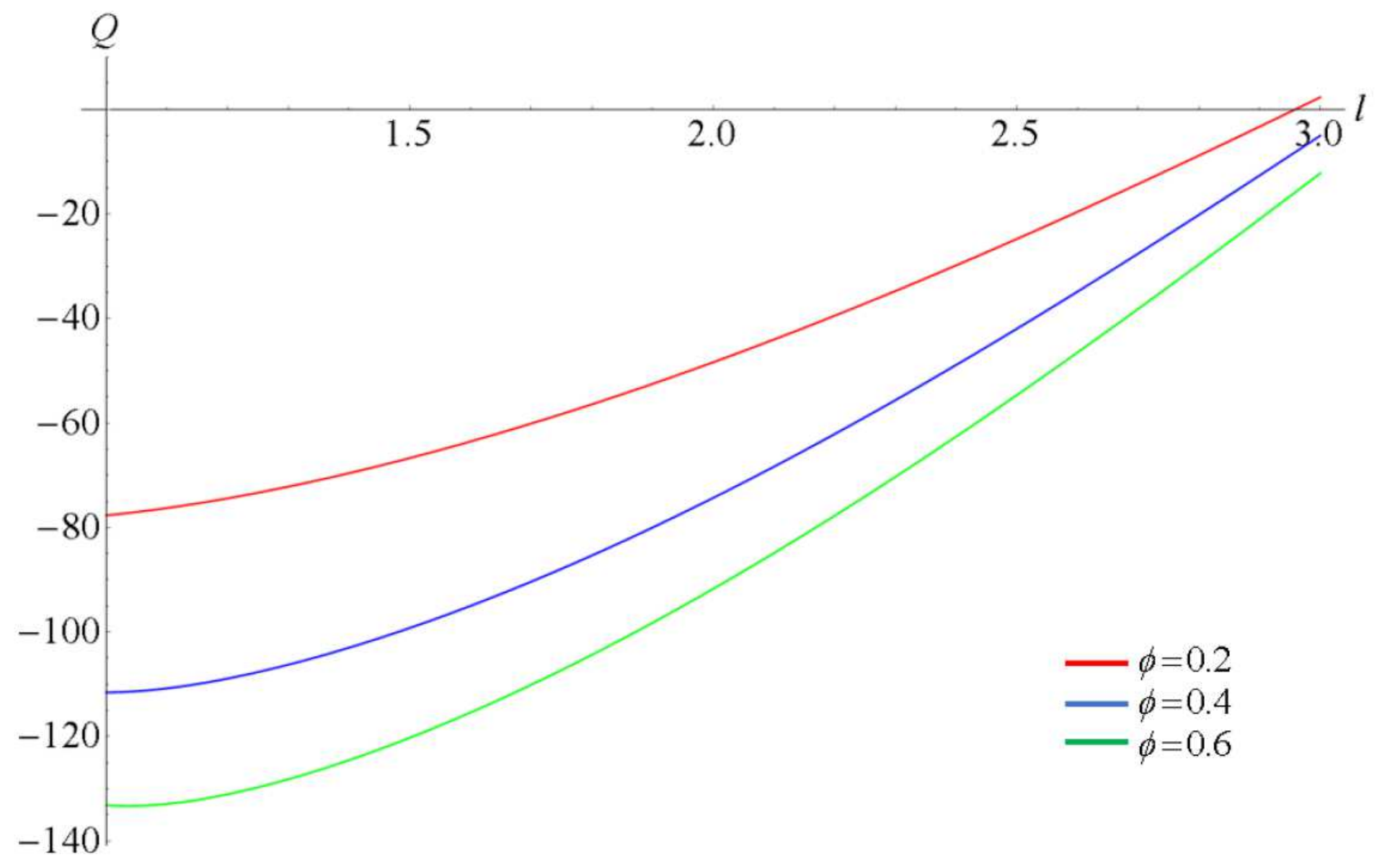

Figure 3

Variation of flow rate with porosity $\varphi$. 


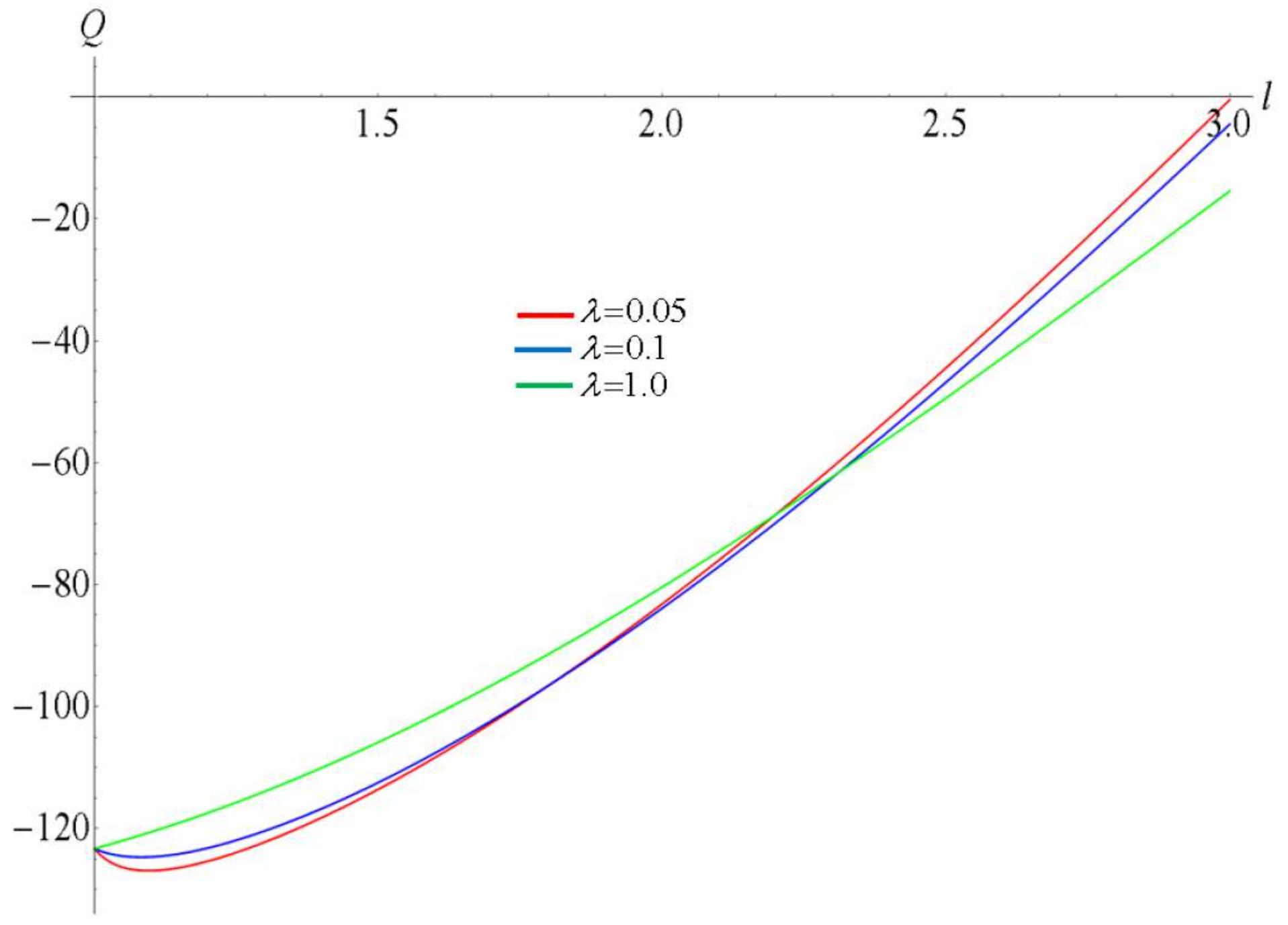

Figure 4

Variation of flow rate with viscosity ratio parameter $\lambda$. 


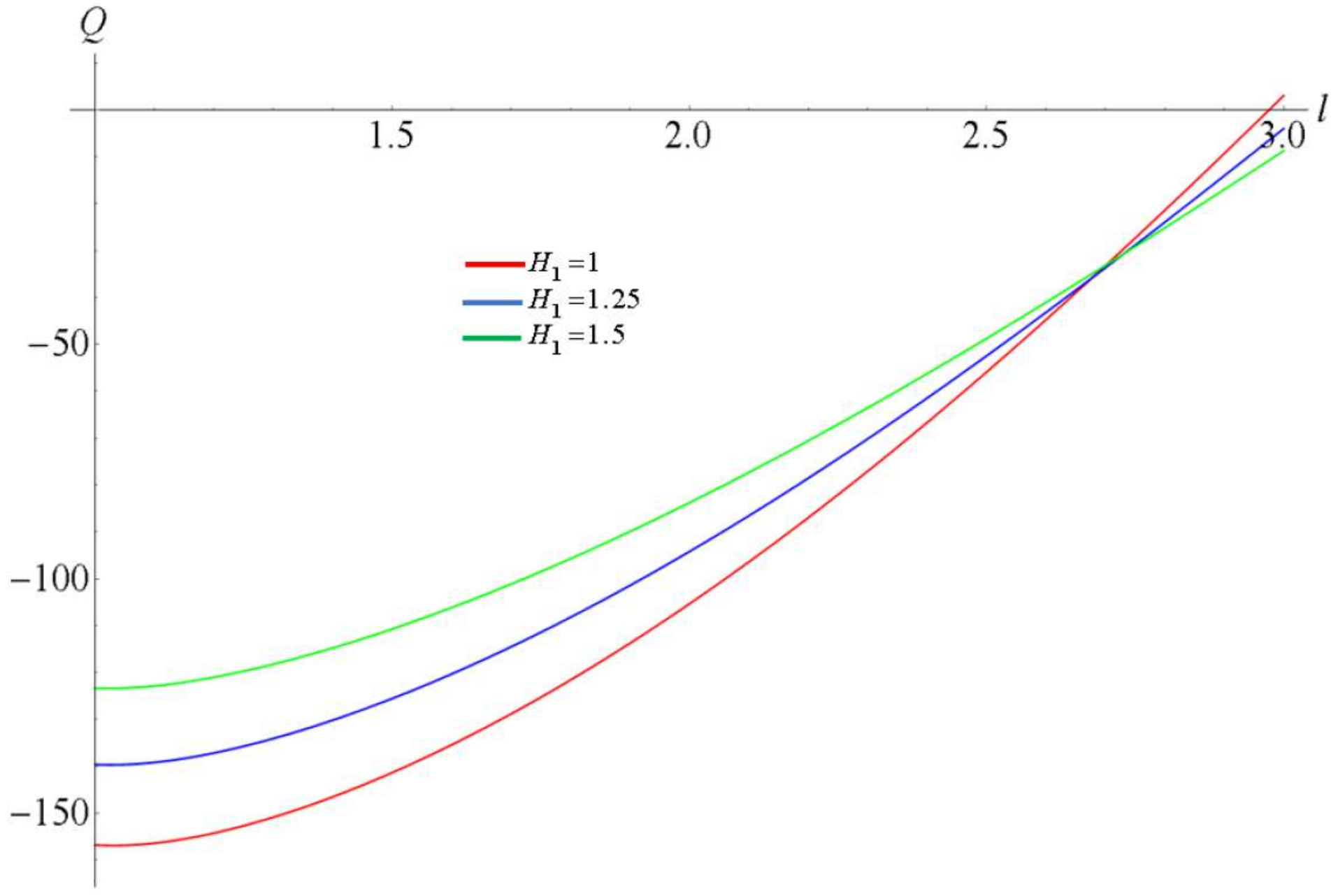

Figure 5

Variation of flow rate with Hartmann number $\mathrm{H} 1$. 


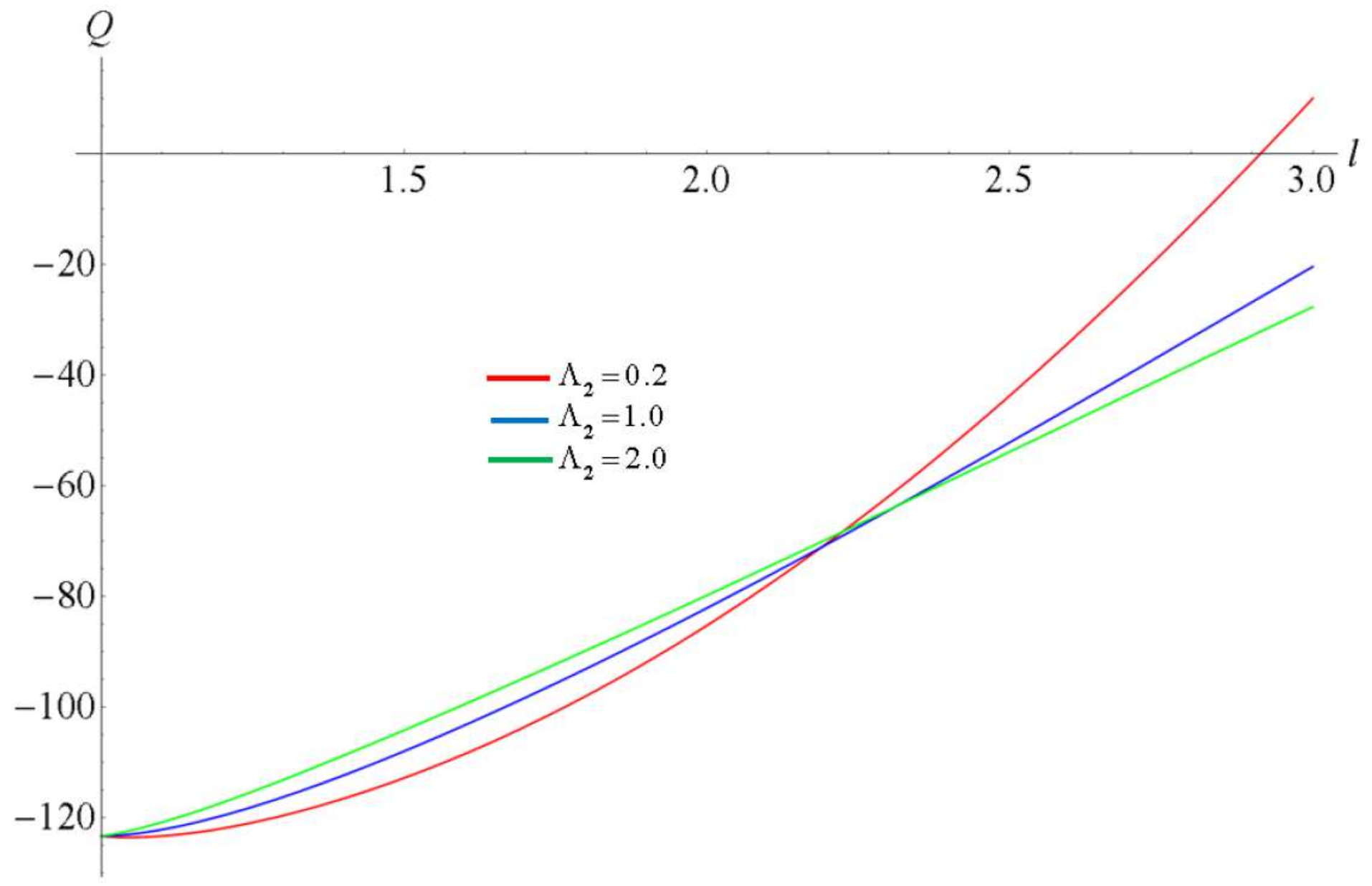

Figure 6

Variation of flow rate with conductivty ratio parameter $\Lambda 2$. 


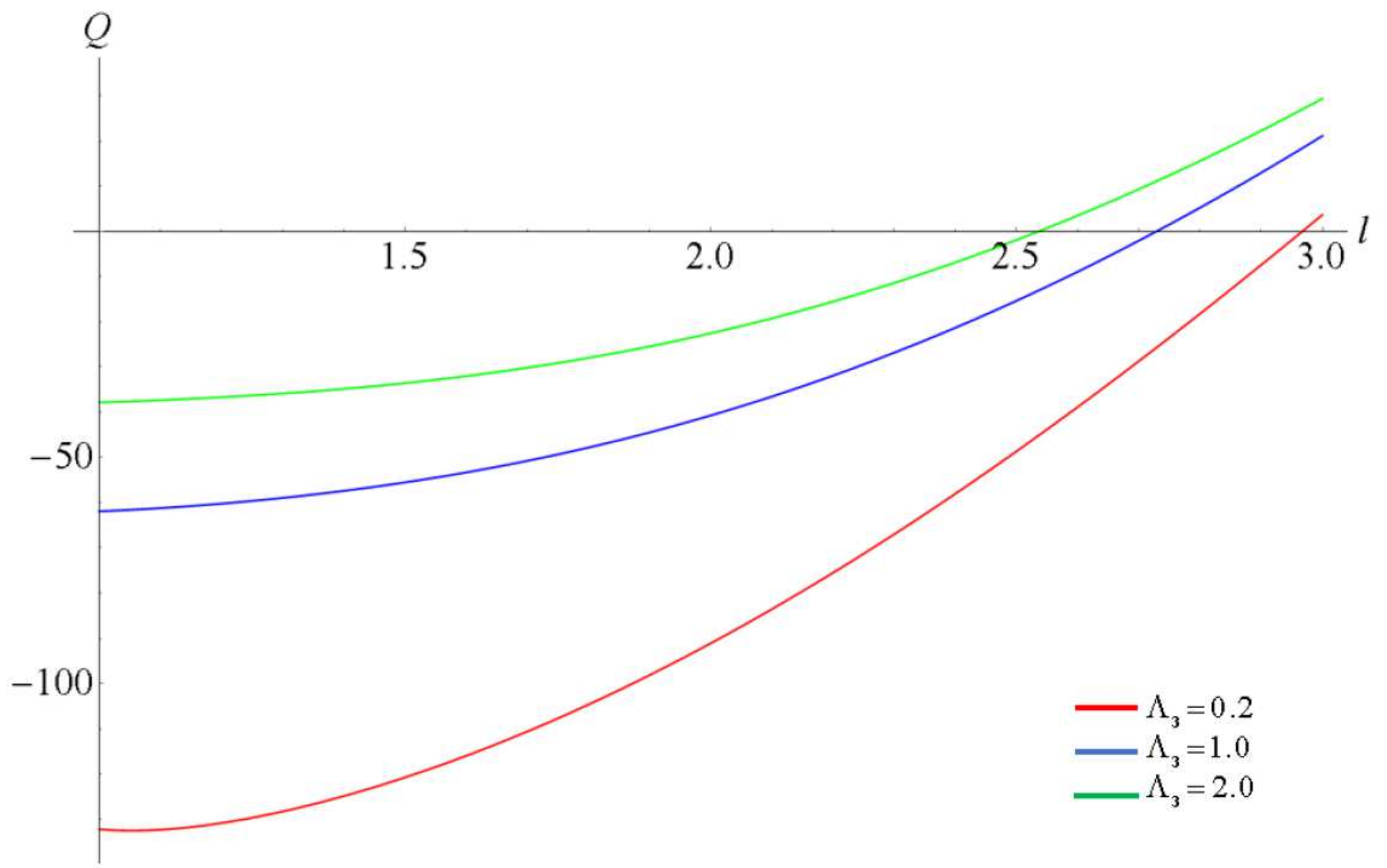

Figure 7

Variation of flow rate with conductivty ratio parameter $\Lambda 3$. 


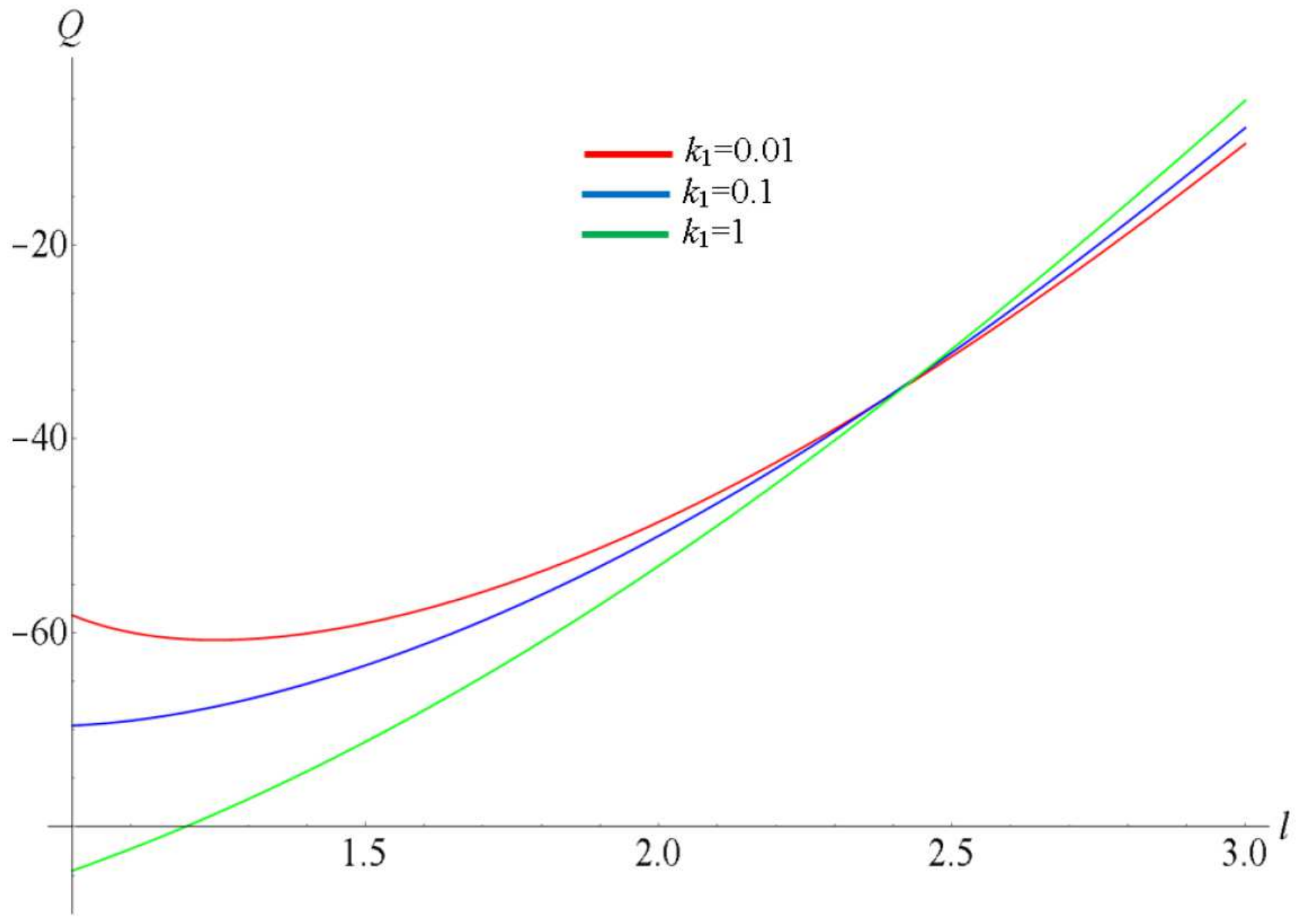

Figure 8

Variation of flow rate with Darcy number k1. 


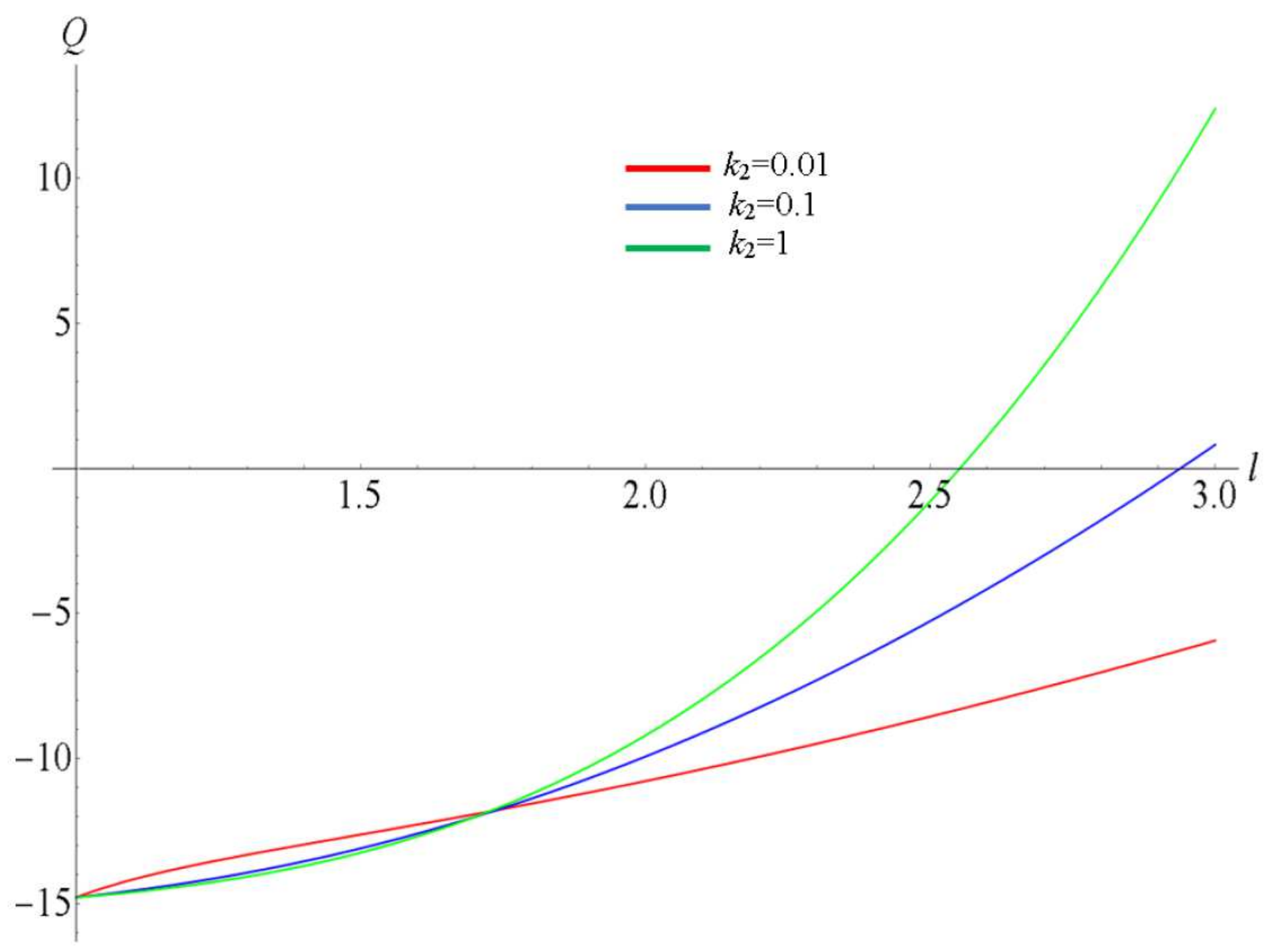

Figure 9

Variation of flow rate with Darcy number k2. 


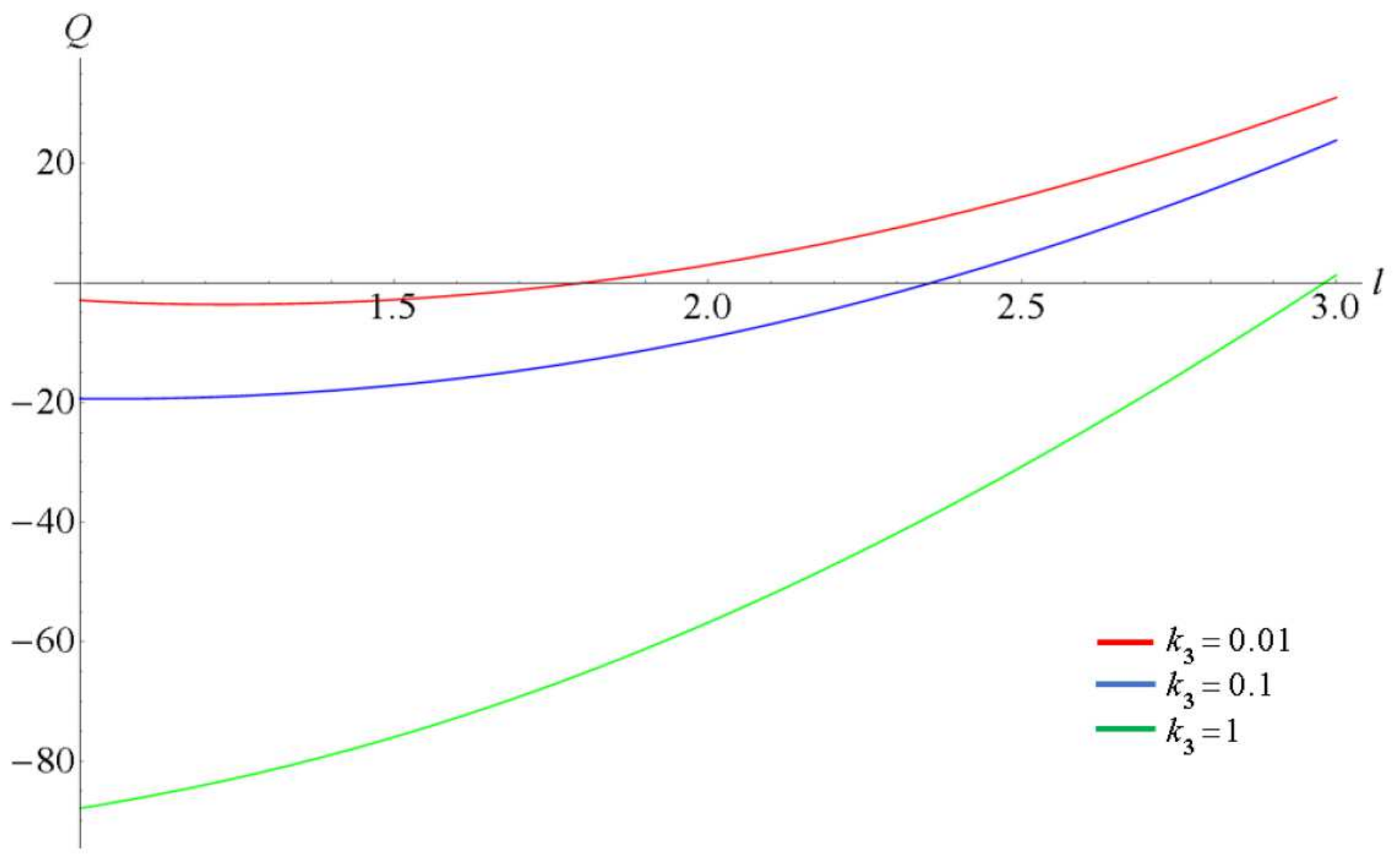

Figure 10

Variation of flow rate with Darcy number k3. 


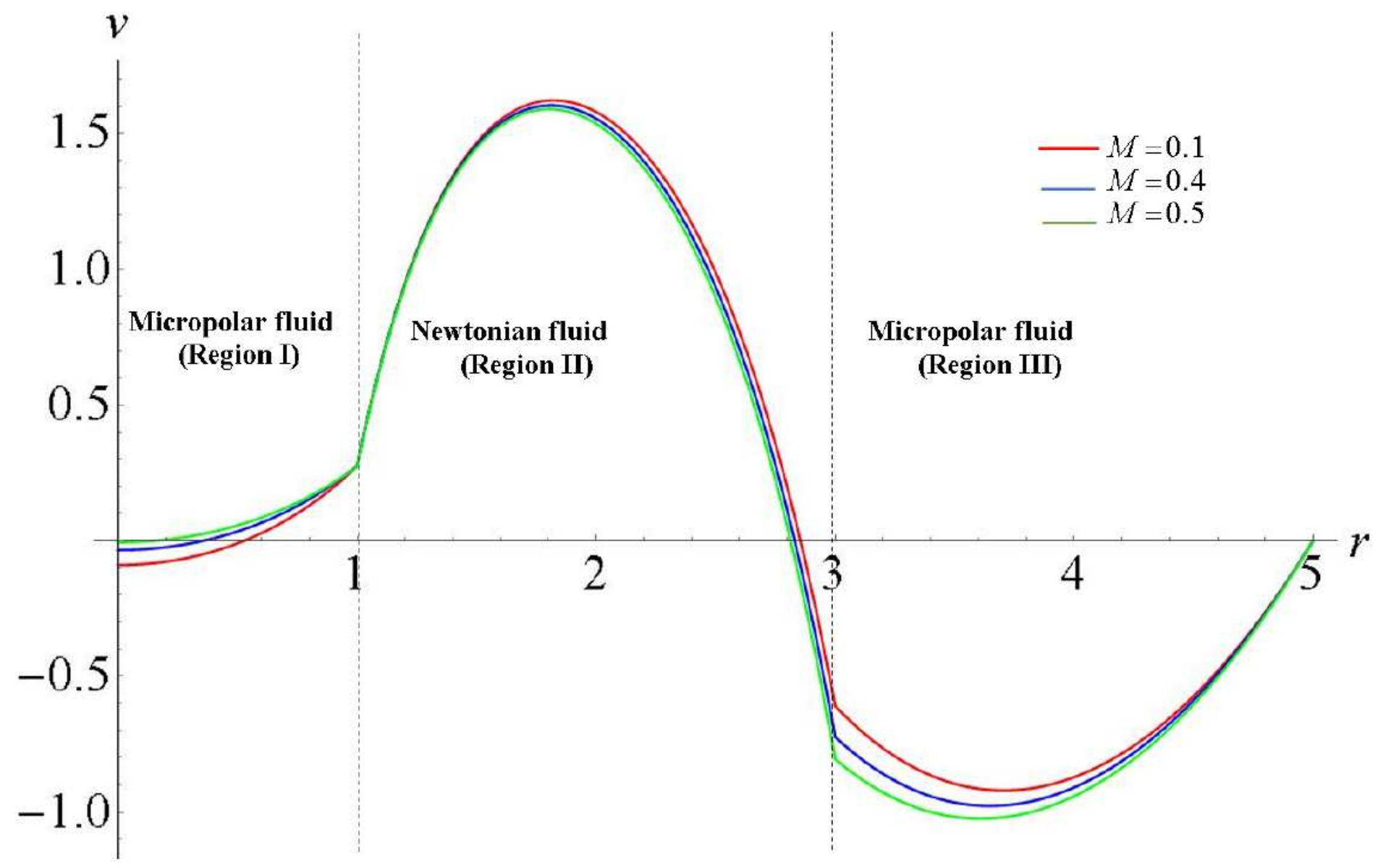

Figure 11

Variation of fluid velocity with micropolar parameter $\mathrm{M}$. 


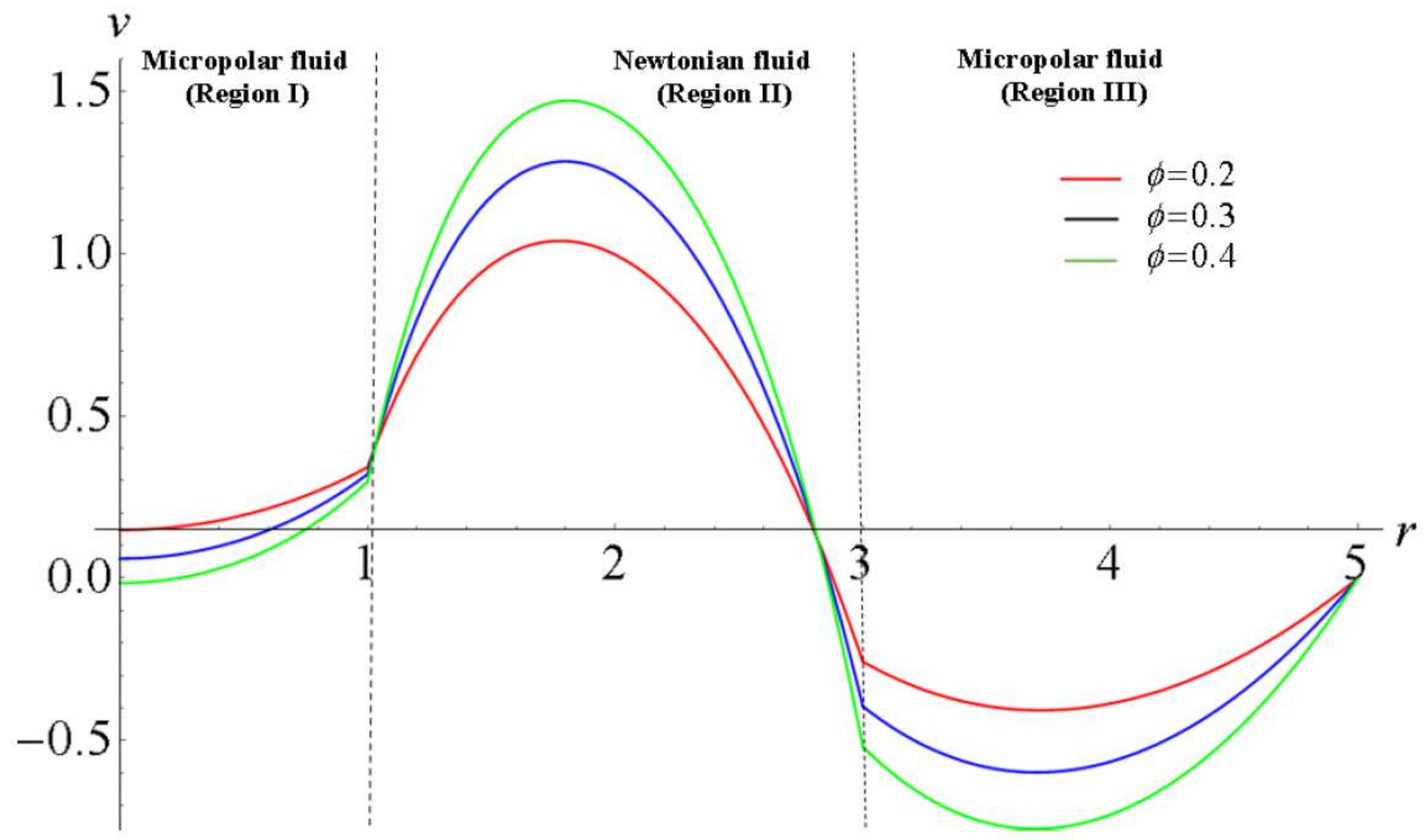

Figure 12

Variation of fluid velocity with porosity $\varphi$. 


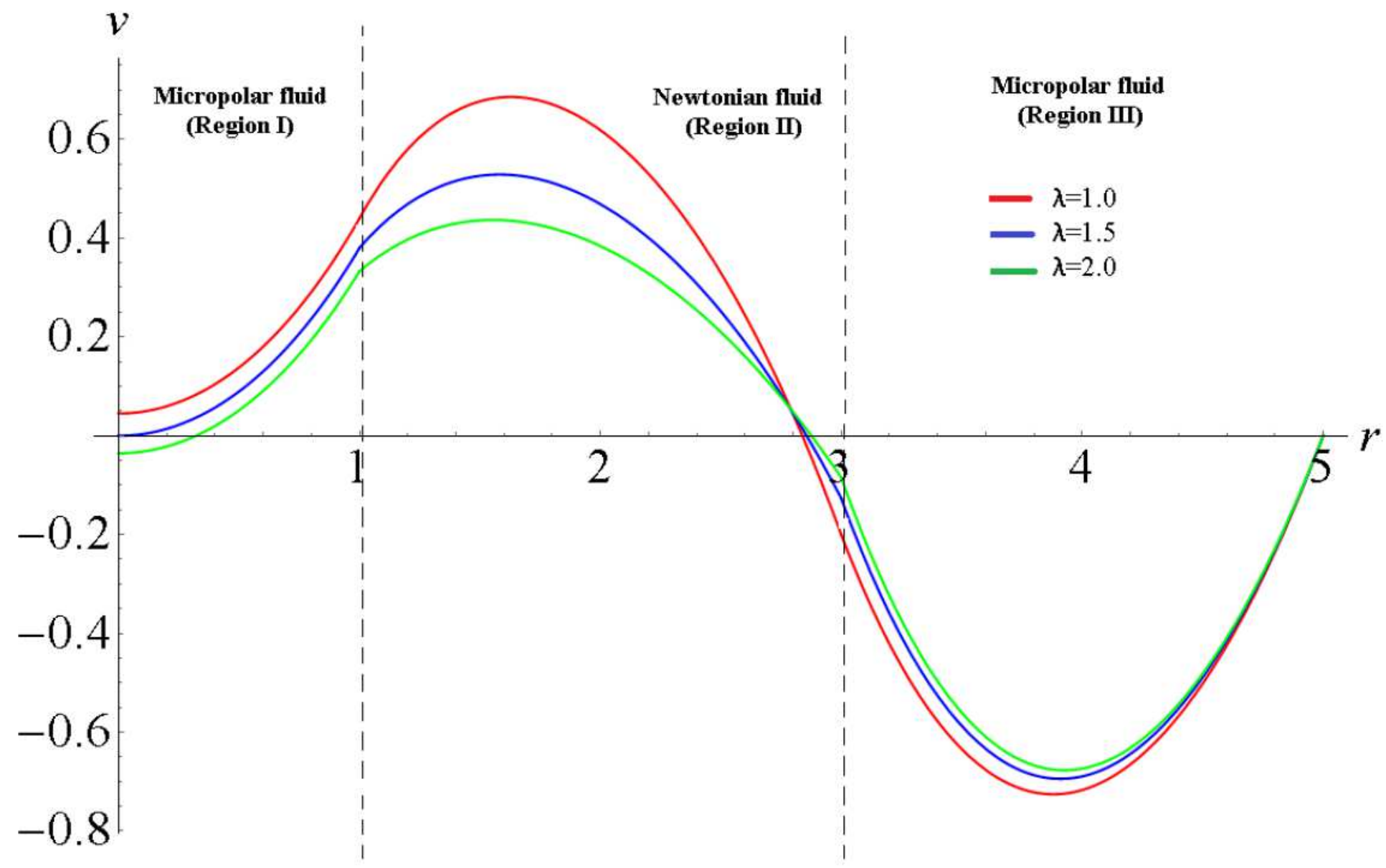

Figure 13

Variation of fluid velocity with viscosity ratio $\lambda$. 


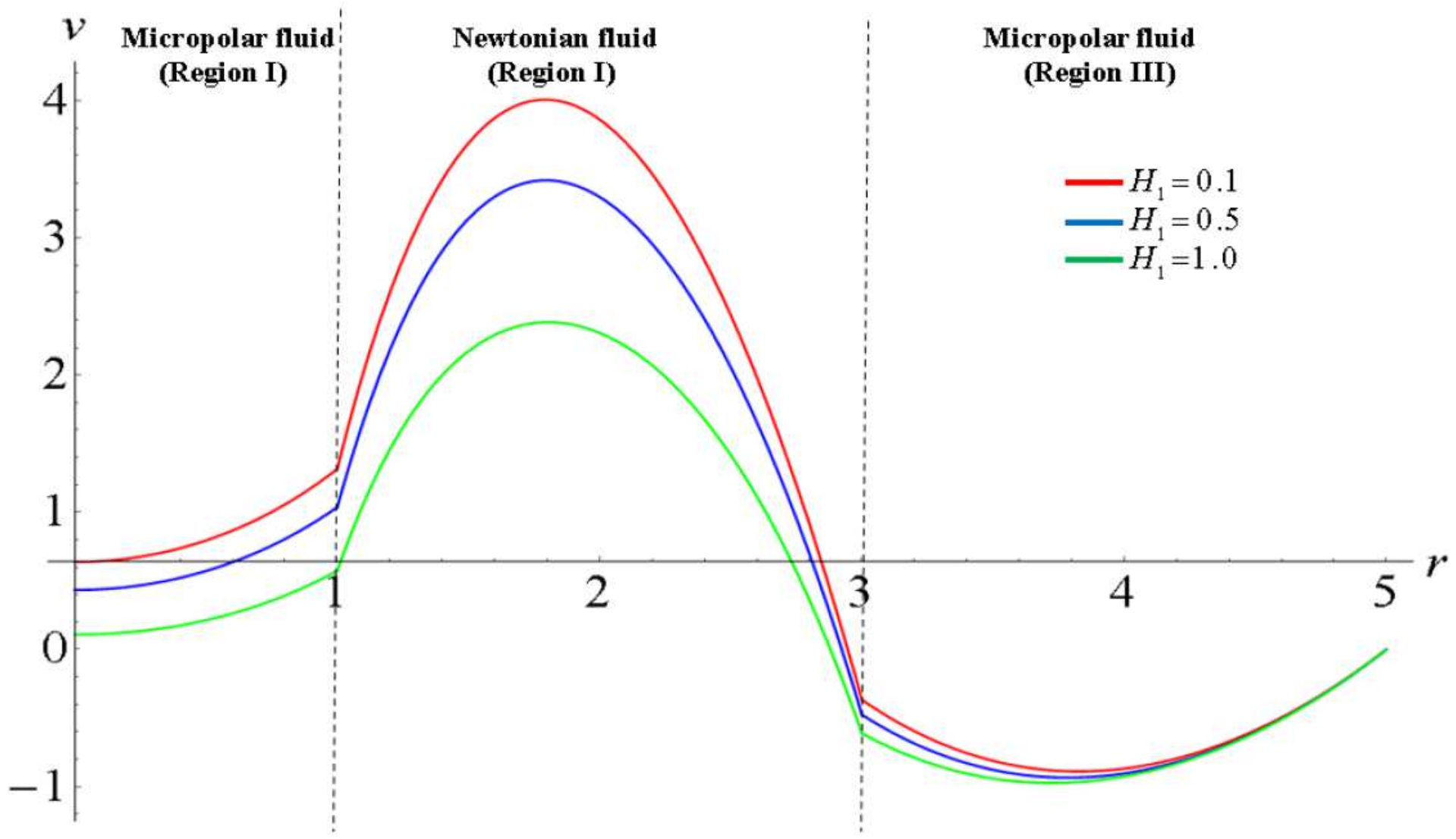

Figure 14

Variation of fluid velocity with Hartmann number $\mathrm{H} 1$. 


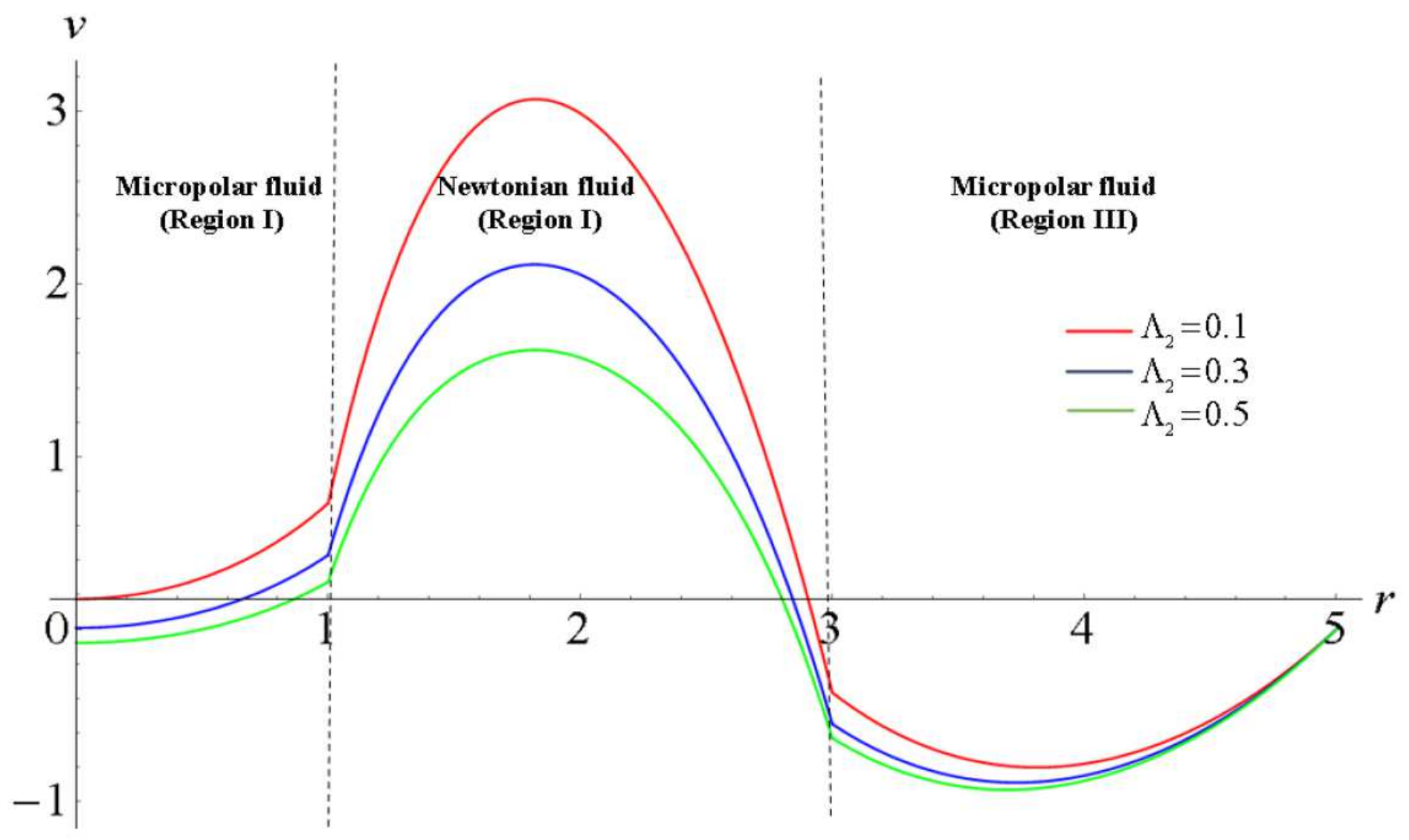

Figure 15

Variation of fluid velocity with conductivity ratio parameter $\Lambda 2$. 


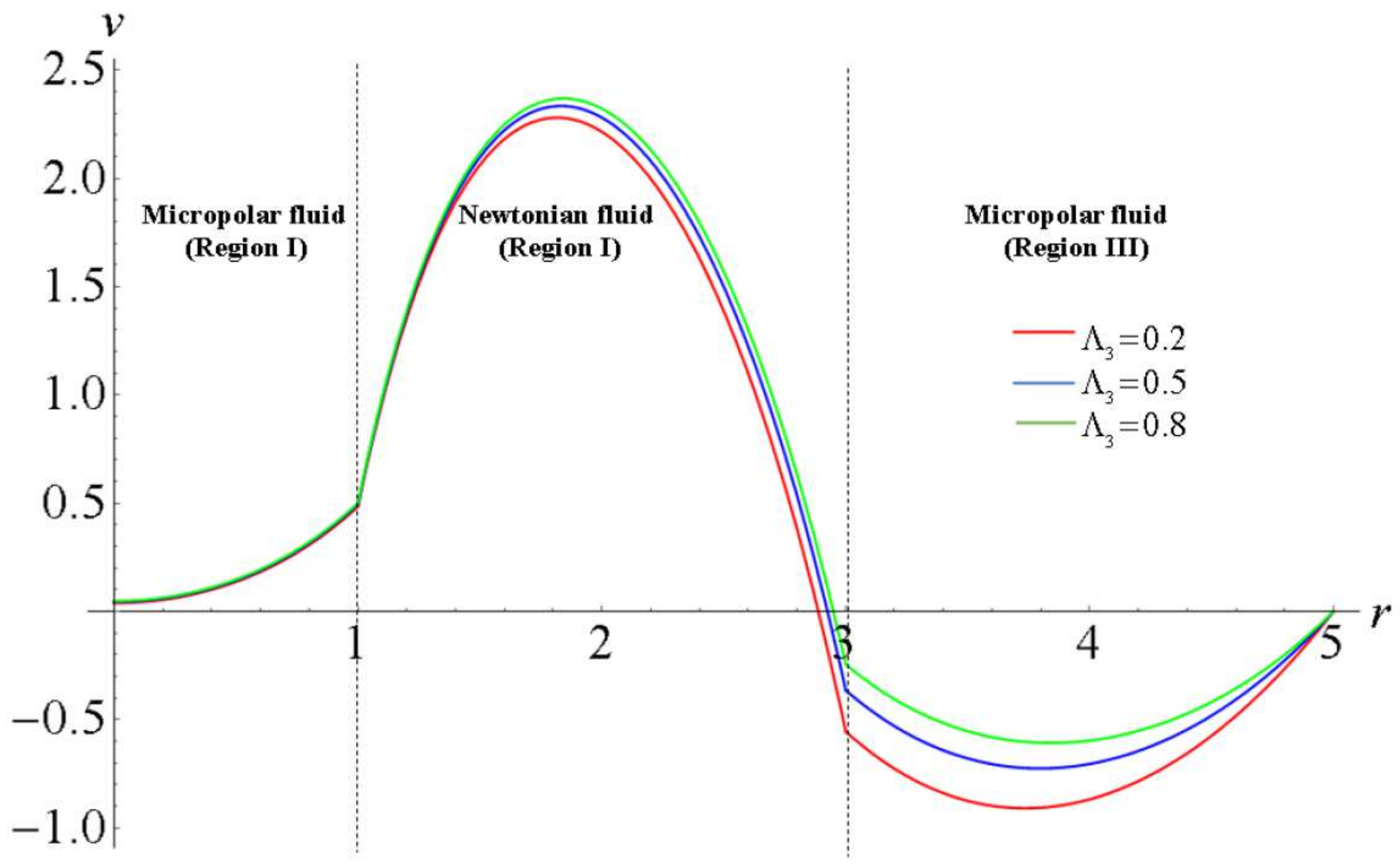

Figure 16

Variation of fluid velocity with conductivity ratio parameter $\wedge 3$. 


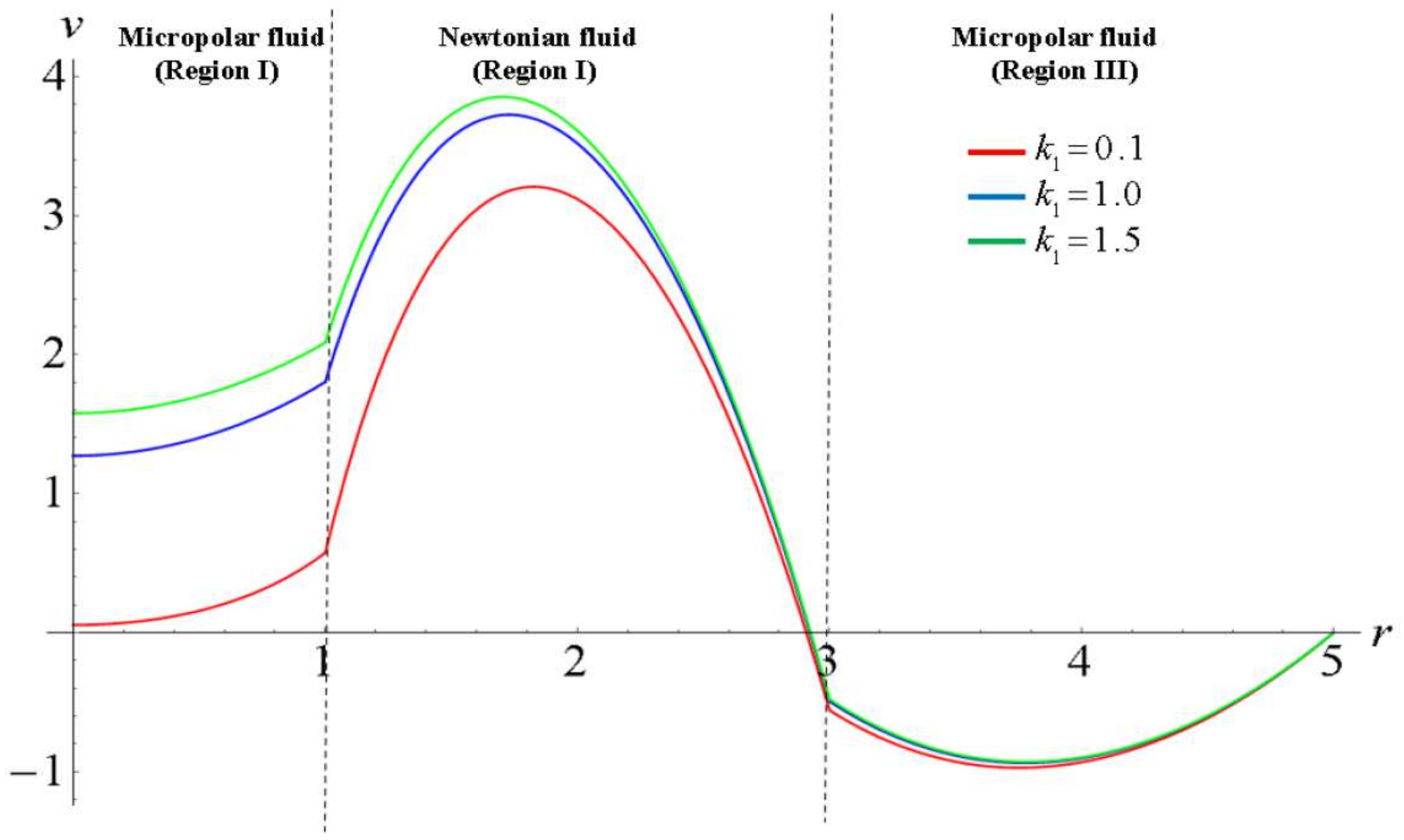

Figure 17

Variation of fluid velocity with Darcy number k1. 


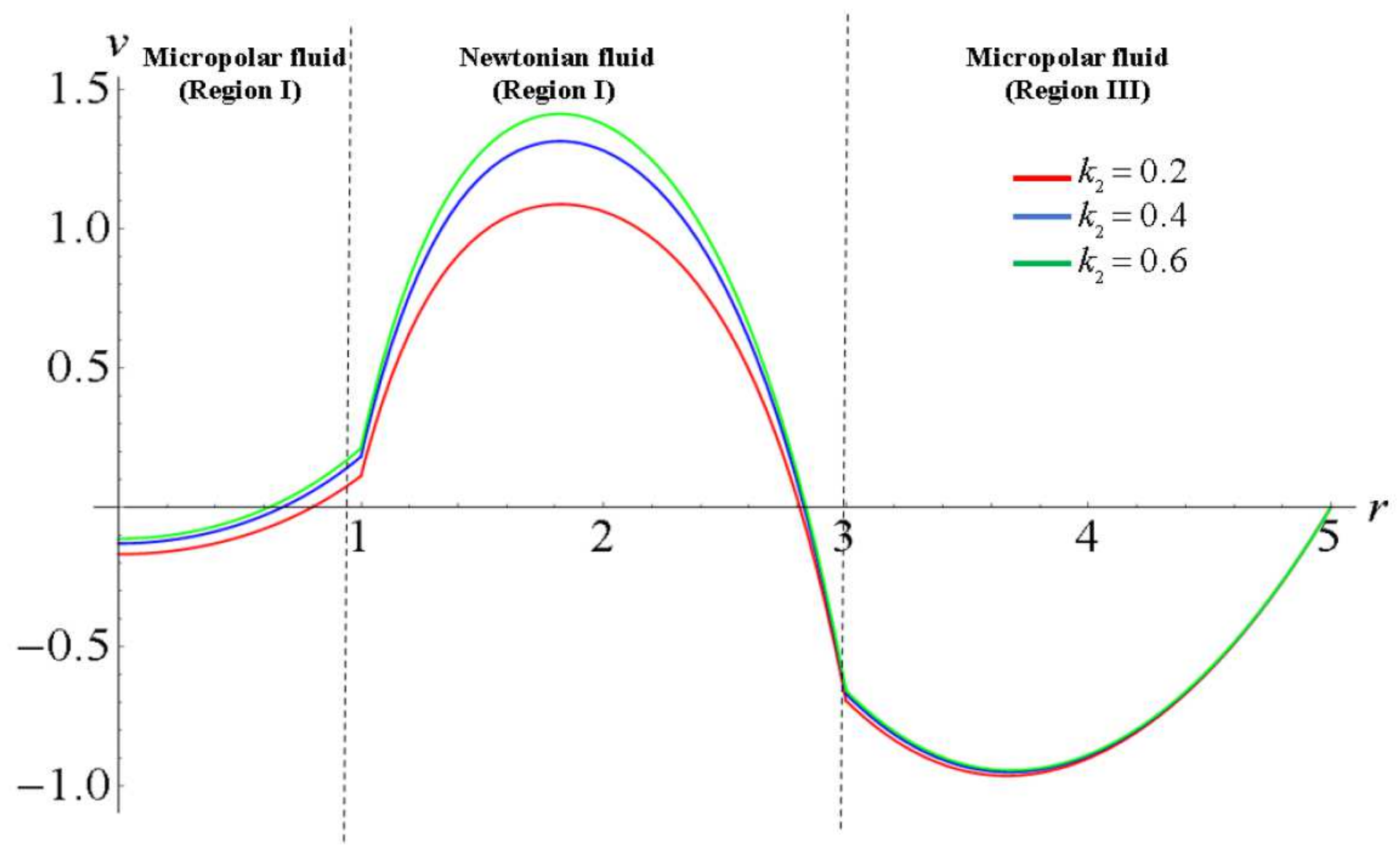

Figure 18

Variation of fluid velocity with Darcy number k2.

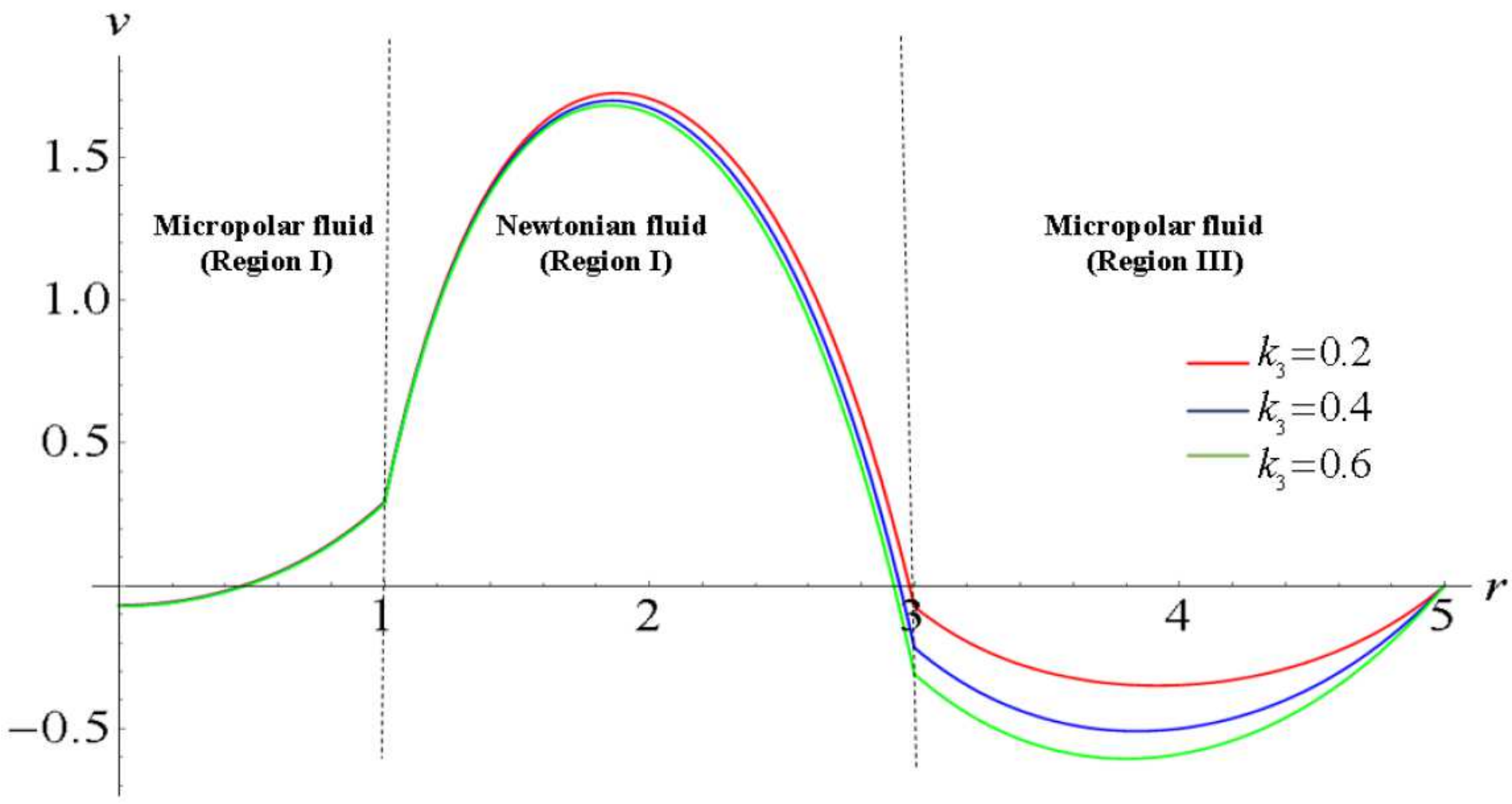


Figure 19

Variation of fluid velocity with Darcy number k3. 